August 2001

\title{
THE PRINCIPLE OF SYMMETRIC CRITICALITY IN GENERAL RELATIVITY
}

\author{
MARK E. FeLS \\ Department of Mathematics and Statistics \\ Utah State University, Logan, Utah 84322 \\ Charles G. Torre \\ Department of Physics \\ Utah State University, Logan, Utah 84322
}

\begin{abstract}
We consider a version of Palais' Principle of Symmetric Criticality (PSC) that is applicable to the Lie symmetry reduction of Lagrangian field theories. PSC asserts that, given a group action, for any group-invariant Lagrangian the equations obtained by restriction of Euler-Lagrange equations to group-invariant fields are equivalent to the Euler-Lagrange equations of a canonically defined, symmetryreduced Lagrangian. We investigate the validity of PSC for local gravitational theories built from a metric. It is shown that there are two independent conditions which must be satisfied for PSC to be valid. One of these conditions, obtained previously in the context of transverse symmetry group actions, provides a generalization of the well-known unimodularity condition that arises in spatially homogeneous cosmological models. The other condition seems to be new. The conditions that determine the validity of PSC are equivalent to pointwise conditions on the group action alone. These results are illustrated with a variety of examples from general relativity. It is straightforward to generalize all of our results to any relativistic field theory.
\end{abstract}




\section{Introduction}

An important approach to studying properties of the solution space of gravitational field equations is to restrict attention to metrics and matter fields that admit a specified group of symmetries. It was noted quite some time ago by Hawking [1], and subsequently discussed in some detail by MacCallum and Taub [2], that with homogeneous cosmological models ("Bianchi models") one cannot always impose the symmetry on the fields in the Einstein-Hilbert action functional since varying the action in this restricted class of fields may not yield the correct field equations. In particular, it was noted that only the Bianchi class A groups would, in general, allow for a successful symmetry reduction of the EinsteinHilbert action. Many others have elaborated on this issue in homogeneous cosmology, see, for example, $[3,4,5,6,7,8]$ and references therein. As Hawking points out in [1], the difficulty which arises with the Bianchi class B models is due to the presence of a non-trivial boundary term in the restricted variational principle. Such a difficulty does not appear in many other symmetry reductions. For example, Pauli restricts the Einstein-Hilbert action to a class of static, spherically symmetric metrics and obtains the reduced Einstein equations by Hamilton's principle [9] (he attributes this approach to Weyl). In addition, Lovelock has shown that a variety of Lagrangians for fourth-order field equations allow for reduction by spherical symmetry [10]. In light of such examples, it is natural to ask whether there exist general criteria that allow one to decide for a given symmetry group when one can successfully reduce a generic Lagrangian or action functional. Our goal in this paper is to give a systematic account of the symmetry reduction of gravitational Lagrangians and field equations, and to completely characterize the symmetry group actions that guarantee the reduced Lagrangian produces the reduced field equations.

Viewing these issues strictly from the point of view of action functionals and variational principles Palais has arrived at the Principle of Symmetric Criticality (PSC) [11]. Given a group action on a space of fields, one can consider the restriction of an action functional $S$ to the group invariant fields to obtain the reduced action $\hat{S}$. Palais' PSC asserts that, for any group invariant functional $S$, critical points of $\hat{S}$ within the class of group invariant fields are (group invariant) critical points of $S$. As Palais emphasized, PSC need be neither well-defined nor valid. Under hypotheses that guarantee PSC makes sense, he goes on to give necessary and sufficient conditions for the validity of PSC in a variety of settings. Unfortunately, a straightforward application of these results to general relativity (and, more generally, classical field theory) is somewhat awkward since one must decide at the outset what class of spacetimes to consider in the variational principle, what asymptotic and/or boundary conditions to impose, what to do about spacetime singularities, etc. Moreover, different group actions may necessitate different choices in this regard. All these issues, which are fundamentally global in nature, will arise when using PSC formulated in terms of the action integral, viewed as a functional on the infinite-dimensional space of 
metrics.

These difficulties can be avoided by using a purely local formulation of PSC that is based on the Lagrangian rather than on the action integral. The version of PSC adopted in this paper asserts that, for a given group action and for any group invariant Lagrangian, the reduced field equations obtained by restriction of Euler-Lagrange equations to group invariant fields are equivalent to the Euler-Lagrange equations of a canonically defined, reduced Lagrangian. This formulation of PSC was studied in [12] under the hypothesis of a transverse symmetry group action. Using tools developed in [13], this formulation of PSC has been extended to the general, non-transverse case in [14]. As we shall see, by using a purely local formulation of PSC based upon the Lagrangian, it is possible to give necessary and sufficient conditions for the validity of PSC once and for all, without having to wrestle with the complications mentioned in the previous paragraph.

In the context of an arbitrary metric theory of gravity in any number of dimensions, we shall show that there are two independent conditions which determine the validity of PSC. One of these already appears for transverse group actions in [12]; it generalizes the restriction to Bianchi class A in the case of homogeneous cosmological models, which was noted above. The second condition for PSC is only relevant when considering non-transverse group actions [14] and does not seem to have been treated in the physics literature on symmetry reduction of variational principles. The conditions that determine the validity of PSC are equivalent to pointwise conditions on the group action. Thus, the validity of PSC is determined solely by the group action, irrespective of the Lagrangian, the spacetime manifold, or the choice of asymptotic or boundary conditions. It is straightforward to generalize these results to other settings, e.g., a field theory that includes other fields besides the metric, or for a theory of matter fields on a fixed spacetime.

We check the validity of PSC for a number of examples from general relativity. The group actions used in these examples have largely been taken from [15]. There, Petrov has provided a (not quite complete) classification of Killing vector fields in four dimensions. It is a simple exercise to check PSC for any of the vector field systems appearing in [15] by following the pattern of the examples presented here.

It should be emphasized at the outset that the symmetry reductions we consider do not involve any "coordinate conditions" or "gauge fixing conditions". In what follows we will always work with the most general metrics admitting the chosen isometry groups, and it is in this setting that we discuss the validity of PSC. Of course, if metric components are eliminated from the Lagrangian using coordinate conditions one will not, in general, recover the corresponding Euler-Lagrange equations. It is interesting to note, however, that for certain symmetry reductions there exist privileged classes of coordinate conditions which can be imposed in the Lagrangian without causing a net loss of independent reduced field equations. See $[9,10]$ for illustrations of this phenomenon. 
This paper is organized as follows. Section 2 summarizes the prerequisites from the theory of isometry groups and the construction of group invariant metrics. Section 3 defines group-invariant Lagrangians and field equations. Section 4 details the construction of the reduced equations and reduced Lagrangian. Section 5 gives a precise formulation of PSC and derives the necessary and sufficient conditions for its validity. Section 6 provides a variety of examples that illustrate the results presented in the previous sections. Appendix A provides a brief description of the generalization of our results to the case where other types of fields are included, either coupled dynamically to the metric or propagating on a fixed spacetime. Appendix B gives a precise notion of equivalence of differential equations which we use to formulate PSC.

\section{Metrics with Symmetry}

Often in general relativity one fixes a spacetime $(M, g)$ and determines its isometry group (or algebra), e.g., by solving the Killing equations for the Killing vector fields. However, when considering symmetry reduction of a gravitational theory we take the opposite point of view and are interested in restricting attention to all spacetimes which admit a chosen isometry group (or algebra). In order to parameterize the spacetime metrics which admit a specified isometry group we identify a bundle over $M / G$ whose sections are in one to one correspondence with the sought after spacetimes. In this section we describe the construction of this bundle. A short summary of the infinitesimal version of the problem is also given at the end of the section. Further details can be found in [13].

Let $\mathcal{S}$ be the space of smooth symmetric tensor fields of type $\left(\begin{array}{l}0 \\ 2\end{array}\right)$ on the manifold $M$, and denote by $\mathcal{Q} \subset \mathcal{S}$ the subset of smooth Lorentz metrics. Let

$$
\mu: G \times M \rightarrow M,
$$

be an action of the Lie group $G$ on $M$. The group $G$ acts naturally on $\mathcal{S}$ and $\mathcal{Q}$ by pull-back:

$$
g \rightarrow \mu_{\gamma}^{*} g, \quad g \in \mathcal{S}, \quad \gamma \in G
$$

where $\mu_{\gamma}: M \rightarrow M$ is the diffeomorphism obtained by restricting $\mu$ in (2.1) to a fixed element $\gamma \in G$. A symmetric tensor $g \in \mathcal{S}$ is $G$-invariant if

$$
\mu_{\gamma}^{*} g=g \quad \forall \gamma \in G .
$$

Let $\mathcal{S}^{G} \subset \mathcal{S}$ be the subset of $G$-invariant symmetric $\left(\begin{array}{l}0 \\ 2\end{array}\right)$ tensor fields on $M$, and let $\mathcal{Q}^{G} \subset \mathcal{S}^{G}$ the $G$-invariant metrics. Thus each $g \in \mathcal{Q}^{G}$ admits $G$ as an isometry group.

The passage from $\mathcal{Q}$ to $\mathcal{Q}^{G}$ according to the $G$-invariance condition (2.2) involves two types of "reduction". First of all, there is the familiar "dimensional reduction" in the 
number of independent variables from the spacetime dimension to the codimension of the orbits of the group in $M$. Thus the set of $G$-invariant metrics can be parametrized by fields on the reduced spacetime $M / G$. Henceforth we assume the quotient space $M / G$ is a smooth manifold. The second type of reduction determines the number of independent components of the metric (or fields) that are essential in parametrizing $\mathcal{Q}^{G}$. The procedure which determines the reduction of the metric components will be used throughout the article so we present some details.

Let $G$ be a Lie group acting on $M$, the isotropy group $G_{x}$ of a point $x \in M$ is the subgroup

$$
G_{x}=\{\gamma \in G \quad \mid \mu(\gamma, x)=x\} .
$$

If the isotropy group $G_{x}$ has dimension $p$ and the group itself has dimension $d$, then the orbit through $x$ has dimension $l=d-p$. The isotropy group $G_{x}$ acts on the tangent space $T_{x} M$ at $x$ by the push-forward map $\mu_{\gamma *}: T_{x} M \rightarrow T_{x} M$. That is, given $\gamma \in G_{x}$ and $V \in T_{x} M$, then

$$
(\gamma, V) \rightarrow \mu_{\gamma *} V
$$

The homomorphism $\gamma \rightarrow \mu_{\gamma *}$ of $G_{x} \rightarrow G L\left(T_{x} M\right)$ is the linear isotropy representation of the group $G_{x}$. Consequently there is the induced representation of $G_{x}$ on the tensor algebra $\otimes\left(T_{x} M\right)$ and its dual. On the space $S_{x}=T_{x}^{*} M \odot T_{x}^{*} M$ of symmetric $\left(\begin{array}{l}0 \\ 2\end{array}\right)$ tensors at $x$, the representation is

$$
\left(\gamma, g_{x}\right) \rightarrow \mu_{\gamma^{-1}}^{*} g_{x}, \quad \gamma \in G_{x}, g_{x} \in S_{x} .
$$

Suppose $g \in \mathcal{S}^{G}$, then by (2.2) the linear isotropy representation (2.3) on $g_{x}$ gives

$$
\mu_{\gamma^{-1}}^{*} g_{x}=g_{x} .
$$

Thus if $g \in \mathcal{S}^{G}$ then $g_{x} \in S_{x}^{G x}$, the fixed points of the linear isotropy representation. Equation (2.4) is the fundamental constraint on the values which any $g \in \mathcal{S}^{G}$ can take and is the second type of reduction mentioned above.

The set $\mathcal{Q}$ is the space of sections of the bundle $Q$ whose fiber is $Q_{x} \subset T_{x}^{*} M \odot T_{x}^{*} M$, the set of Lorentz-signature quadratic forms on $T_{x} M$. Using (2.4) we define the subset $K_{x} \subset Q_{x}$ to be

$$
K_{x}=Q_{x}^{G x}=\left\{g_{x} \in Q_{x} \quad \mid \quad \mu_{\gamma^{-1}}^{*} g_{x}=g_{x}, \quad \forall \gamma \in G_{x}\right\} .
$$

$K_{x}$ is the set of the Lorentz-signature quadratic forms on $T_{x} M$ which are invariant with respect to the action of $G_{x}$ on $Q_{x}$. Now let

$$
K=\bigcup_{x \in M} K_{x}
$$


We assume that $K \subset Q$ is a fixed rank sub-bundle of $Q$, then by (2.4) if $g \in \mathcal{Q}^{G}$ then $g: M \rightarrow K$ is a section of $\pi: K \rightarrow M$.

Condition (2.4) is only a necessary condition for $g \in \mathcal{Q}^{G}$, and so not every section of $K$ is a $G$-invariant tensor field. One more step is required to determine the bundle over $M / G$ whose sections parameterize $\mathcal{Q}^{G}$. Since $G$ acts transversely to the fibers of $K$ [16], the quotient $K / G$ is a bundle $\hat{K} \rightarrow M / G$ with the same fiber as $K$ but with base space $M / G$. Each $G$-invariant metric $g \in \mathcal{Q}^{G}$ determines a section $\hat{q}$ of the bundle $\hat{K}$, and each section $\hat{q}$ of $\hat{K}$ determines a $G$-invariant metric $g \in \mathcal{Q}^{G}[13]$. Thus there exists a bijection,

$$
\Phi: \hat{\mathcal{Q}} \rightarrow \mathcal{Q}^{G}
$$

identifying the space $\mathcal{Q}^{G}$ of $G$-invariant metrics on $M$ with the space of sections, $\hat{\mathcal{Q}}$, of $\hat{K}$. Using the bijection $\Phi$, the set $\mathcal{Q}^{G}$ is thus parametrized by $m$ functions of $r$ variables, $\hat{q} \in \hat{\mathcal{Q}}$, where $m$ is the dimension of the solution space to $(2.4)$ and $r=\operatorname{dim}(M / G)$. We let $g(\hat{q})=\Phi(\hat{q})$ be the $G$-invariant metric defined by $\hat{q}{ }^{*}$

Throughout we make the blanket hypothesis that a parallel construction arises for the parametrization of $G$-invariant tensor fields of any type. In particular, we assume that there is a vector space of fields $\hat{\mathcal{S}}$ on $M / G$, which are sections of a vector bundle over $M / G$ and which parametrize the vector space $\mathcal{S}^{G}$ of $G$-invariant symmetric $\left(\begin{array}{l}0 \\ 2\end{array}\right)$ tensor fields on $M$. The corresponding bijection is denoted by

$$
P: \hat{\mathcal{S}} \rightarrow \mathcal{S}^{G}
$$

where $P$ is a linear differential operator of order 0 . We note that

$$
\mathcal{Q}^{G} \subset \mathcal{S}^{G}, \quad \hat{\mathcal{Q}} \subset \hat{\mathcal{S}}
$$

and

$$
\Phi=\left.P\right|_{\hat{\mathcal{Q}}}
$$

Let $G$ act on $M$ and let $\Gamma$ be the Lie algebra of infinitesimal generators of the action of $G$. If $g \in \mathcal{S}^{G}$ then $g$ satisfies the infinitesimal invariance equations

$$
L_{X} g=0, \quad \forall X \in \Gamma,
$$

which are the Killing equations if $g \in \mathcal{Q}^{G}$. Suppose instead of being given $G$ that we are given a Lie algebra of vector-fields $\Gamma$ on an open set $U \subset M$. In general $\Gamma$ will not be the

* For a large class of group actions the space $\hat{\mathcal{Q}}$ can be identified with the following set of Kaluza-Klein fields on $M / G$ : (i) the space of metrics, (ii) a space of connections on a principal bundle, i.e., non-Abelian gauge fields, and (iii) a space of scalar ("Higgs") fields determining a non-linear sigma model. See [17] for details. 
infinitesimal generators of a Lie group acting on $M$ (or $U$ ) so there is no corresponding notion of $G$-invariance. However, $G$-invariance can be replaced by $\Gamma$ invariance as follows.

Let $\mathcal{S}^{\Gamma}$ be the symmetric $\left(\begin{array}{l}0 \\ 2\end{array}\right)$ tensor fields satisfying (2.7) on $U$ and similarly for $\mathcal{Q}^{\Gamma} \subset \mathcal{S}^{\Gamma}$. The parameterization of $\mathcal{Q}^{\Gamma}$ then proceeds along the same lines as $\mathcal{Q}^{G}$. Let $\Gamma_{x_{0}}=\left\{X \in \Gamma \mid X_{x_{0}}=0\right\}$ be the isotropy algebra at $x_{0} \in U$. The condition

$$
\left(L_{X} g\right)_{x_{0}}=0, \quad X \in \Gamma_{x_{0}}
$$

is the infinitesimal version of the isotropy constraint (2.4). The solution to (2.8) determines the reduction of the metric components. The space $M / G$ of reduced dependent variables is replaced by $U / \Gamma$ where points on $U$ are identified if they lie on the same maximal integral manifold of $\Gamma$. A functionally independent set of solutions to $X(f(x))=0, f \in C^{\infty}(U)$ form local coordinates on $U / \Gamma$. If $\Gamma$ is given on a chart $\left(U, x^{\alpha}\right)$ and $X=\xi^{\alpha}(x) \partial_{\alpha} \in \Gamma_{x_{0}}$ then

$$
\left(\xi_{, \beta}^{\alpha} g^{\beta \gamma}+\xi_{, \beta}^{\gamma} g^{\alpha \beta}\right)_{x_{0}}=0
$$

is the isotropy constraint (2.8) which determines the admissible values of $g_{x_{0}}$ for $g \in \mathcal{S}^{\Gamma}$. Finally, we point out that if $G$ is connected then $\mathcal{S}^{G}=\mathcal{S}^{\Gamma}$ where $\Gamma$ is the algebra of infinitesimal generators for the action of $G$ on $M$.

\section{Lagrangians and Field Equations with Symmetry}

A Lagrangian for a metric field theory is a differential operator which, when applied to a metric $g$, yields a spacetime $n$-form $\lambda=\lambda(g)$. Using the first variational formula we have

$$
\delta \lambda=E(\lambda) \cdot \delta g+d \eta(\delta g),
$$

where $\delta g \in \mathcal{S}$ is a symmetric tensor field, and $E(\lambda)$, which is the Euler-Lagrange form of the Lagrangian $\lambda$, is a symmetric $\left(\begin{array}{l}2 \\ 0\end{array}\right)$ tensor-valued $n$-form, and $\eta$ is a linear differential operator mapping any symmetric $\left(\begin{array}{l}0 \\ 2\end{array}\right)$ tensor field $h$ to an $(n-1)$-form $\eta(h)$. Both $E(\lambda)$ and $\eta(\delta g)$ are local functions of the metric and its derivatives. Note that the $(n-1)$-form $\eta(\delta g)$ is only defined up to the addition of an exact $(n-2)$ form locally constructed from the metric, the metric variation, and their derivatives. (The metric variation can only appear linearly.) The Euler-Lagrange equations (or the field equations) are the system of differential equations

$$
E(\lambda)=0
$$

In coordinates $x^{\alpha}$ on $M$ we have

$$
\lambda=L \nu,
$$


where

$$
\nu=d x^{1} \wedge d x^{2} \wedge \cdots \wedge d x^{n}
$$

and

$$
L=L\left(x^{\alpha}, g_{\mu \nu}, g_{\mu \nu, \sigma}, \ldots, g_{\mu \nu, \sigma_{1} \cdots \sigma_{k}}\right)
$$

is a local function of the metric and its derivatives called the Lagrangian density. We have

$$
E(\lambda)=E^{\alpha \beta}(g) \frac{\partial}{\partial x^{\alpha}} \otimes \frac{\partial}{\partial x^{\beta}} \otimes \nu,
$$

with

$$
\begin{gathered}
E^{\alpha \beta}=E^{\beta \alpha}=\frac{\partial L}{\partial g_{\alpha \beta}}-\partial_{\sigma} \frac{\partial L}{\partial g_{\alpha \beta, \sigma}}+\partial_{\sigma_{1}} \partial_{\sigma_{2}} \frac{\partial L}{\partial g_{\alpha \beta, \sigma_{1} \sigma_{2}}}+\cdots+(-1)^{k} \partial_{\sigma_{1}} \cdots \partial_{\sigma_{k}} \frac{\partial L}{\partial g_{\alpha \beta, \sigma_{1} \cdots \sigma_{k}}}, \\
E(\lambda) \cdot \delta g=E^{\alpha \beta} \delta g_{\alpha \beta} \nu,
\end{gathered}
$$

and

$\eta(\delta g)=\left(\eta^{\alpha \beta} \delta g_{\alpha \beta}+\eta^{\alpha \beta, \gamma} \delta g_{\alpha \beta, \gamma}+\cdots+\eta^{\alpha \beta, \gamma_{1} \cdots \gamma_{k-1}} \delta g_{\alpha \beta, \gamma_{1} \cdots \gamma_{k-1}}\right) d x^{1} \wedge d x^{2} \wedge \cdots \wedge d x^{n-1}$.

For example, the Lagrangian we shall use repeatedly in $\S 6$ is the Einstein-Hilbert Lagrangian, which takes the form

$$
\lambda(g)=R(g) \epsilon(g),
$$

where $R$ is the scalar curvature of the metric $g$ and $\epsilon$ is the volume form of $g$. The Euler-Lagrange form is then given by

$$
E(\lambda)=-\mathcal{G} \otimes \epsilon=-\sqrt{|g|} \mathcal{G}^{\alpha \beta} \partial_{\alpha} \otimes \partial_{\beta} \otimes \nu,
$$

where $\mathcal{G}$ is the $\left(\begin{array}{l}2 \\ 0\end{array}\right)$ form of the Einstein tensor. The boundary term $\eta$ can be chosen to be $[18]$

$$
\eta(h)=-*[\operatorname{div} h-d(\operatorname{tr} h)]=-\epsilon_{\alpha \beta \gamma}{ }^{\delta}\left(\nabla^{\gamma} h_{\delta \gamma}-\nabla_{\delta} h_{\gamma}^{\gamma}\right) d x^{\alpha} \wedge d x^{\beta} \wedge d x^{\gamma},
$$

where $\nabla$ is the Levi-Civita connection defined by $g$.

We say that a gravitational theory is $G$-invariant if its Lagrangian $\lambda$ is $G$-equivariant as a map from $\mathcal{Q}$ to the set of $n$-forms on $M$ :

$$
\lambda\left(\mu_{\gamma}^{*} g\right)=\mu_{\gamma}^{*} \lambda(g), \quad \forall \gamma \in G,
$$

that is, $\lambda$ is suitably "covariant" with respect to the symmetry group action. Granted the $G$-equivariance of the Lagrangian, the naturality of the Euler-Lagrange form [19] implies that it is also $G$-equivariant:

$$
E(\lambda)\left(\mu_{\gamma}^{*} g\right)=\mu_{\gamma}^{*} E(\lambda)(g), \quad \forall \gamma \in G .
$$


The $G$-equivariance of the Lagrangian and Euler-Lagrange form guarantees the following key fact: the Lagrangian and the Euler-Lagrange form are $G$-invariant tensor fields when evaluated on a $G$-invariant metric $g \in \mathcal{Q}^{G}$ :

$$
\left.\begin{array}{rl}
\mu_{\gamma}^{*} \lambda(g) & =\lambda(g), \\
\mu_{\gamma}^{*} E(\lambda)(g) & =E(\lambda)(g) .
\end{array}\right\} \forall \gamma \in G, g \in \mathcal{Q}^{G}
$$

We shall in all that follows assume that the field theory under consideration is $G$ invariant. In general relativity and its variants it is normally assumed that the Lagrangian and field equations are in fact equivariant with respect to the whole spacetime diffeomorphism group, that is, the theory is "generally covariant". In this case, for any symmetry reduction the $G$-equivariance of the Lagrangian and field equations is guaranteed since the symmetry group is always acting as a subgroup of the spacetime diffeomorphism group. Nonetheless, we shall not need to assume general covariance in what follows. Thus our results can be applied to all generally covariant (metric) theories of gravity, but also to theories which are not covariant because, e.g., they involve fixed, background fields.

Granted a $G$-equivariant Lagrangian, if we extend the $G$-action to the field variations $\delta g \in \mathcal{S}$, then it follows that $\delta \lambda$ is $G$-equivariant:

$$
\delta \lambda\left(\mu_{\gamma}^{*} g, \mu_{\gamma}^{*} \delta g\right)=\mu_{\gamma}^{*} \delta \lambda(g, \delta g), \quad \forall \gamma \in G .
$$

Because of the $G$-equivariance of the Euler-Lagrange expression it then follows that the volume term $E(\lambda) \cdot \delta g$ and the boundary term $d \eta(\delta g)$ are separately $G$-equivariant. However, it is not immediately clear that the $(n-1)$-form $\eta(\delta g)$ featuring in the boundary term can be chosen to be $G$-equivariant. Iyer and Wald [18] have established the existence of a $G$-equivariant choice of $\eta$ in any generally covariant field theory. More generally, it is possible to establish the existence of a $G$-equivariant choice of $\eta$ for any $G$-invariant field theory admitting a $G$-invariant metric [20]. We assume that such a choice of $\eta$ has been made so that

$$
\eta\left(\mu_{\gamma}^{*} g, \mu_{\gamma}^{*} \delta g\right)=\mu_{\gamma}^{*} \eta(g, \delta g), \quad \forall \gamma \in G .
$$

Then we have that for any $G$-invariant metric $g \in \mathcal{Q}^{G}$ and $G$-invariant metric variation $\delta g \in \mathcal{S}^{G}:$

$$
\left.\begin{array}{c}
\mu_{\gamma}^{*} \delta \lambda=\delta \lambda, \\
\mu_{\gamma}^{*} \eta=\eta
\end{array}\right\} \quad \forall \gamma \in G, g \in \mathcal{Q}^{G}, \delta g \in \mathcal{S}^{G} .
$$

We conclude this section by reminding the reader that the foregoing discussion admits a purely local, infinitesimal description. One simply replaces the group $G$ acting on $M$ with an algebra of vector fields $\Gamma$ on $U \subset M$ and uses the corresponding notions of infinitesimal invariance/equivariance. 


\section{Symmetry Reduction of the Field Equations and Lagrangian}

Given a system of field equations and a group action $(G, \mu)$, we can ask for solutions to the field equations that are $G$-invariant. This simply means that we ask for metrics $g$ that satisfy

$$
E(\lambda(g))=0, \quad \mu_{\gamma}^{*} g=g, \quad \forall \gamma \in G,
$$

that is, we are restricting the field equations from $\mathcal{Q}$ to $\mathcal{Q}^{G}$ :

$$
E(\lambda)(g(\hat{q}))=0 .
$$

The principal idea behind the theory of symmetry reduction is that a system of $G$ equivariant field equations, when restricted to $G$-invariant metrics, is equivalent* to a simpler system of reduced field equations $\hat{\Delta}(\hat{q})=0$ for the fields $\hat{q}$ on $M / G$ that parametrize $\mathcal{Q}^{G}(c f . \S 2)[13]:$

$$
E(\lambda)(g(\hat{q}))=0 \text { on } M \quad \Longleftrightarrow \quad \hat{\Delta}(\hat{q})=0 \text { on } M / G \text {. }
$$

Typically, the equations $\hat{\Delta}=0$ are considerably more tractable than the original field equations since the number of independent and/or dependent variables has been reduced.

One important feature of the reduction from $E(\lambda)=0$ to $\hat{\Delta}=0$ is that, just as the isotropy group (if any) leads to a reduction in the number of independent components of the $G$-invariant metrics, it also reduces the number of independent field equations. This occurs because the field equation tensor $E(\lambda)$, upon restriction to an element of $\mathcal{Q}^{G}$, becomes a $G$-invariant symmetric $\left(\begin{array}{l}2 \\ 0\end{array}\right)$ tensor-valued $n$-form, $E(\lambda)(g(\hat{q}))$, on $M$ and it obeys isotropy constraints at each point in the same way as was discussed in $\S 2$ for the metric. Therefore, provided the group action is not free, the number of reduced field equations $\hat{\Delta}=0$ will be less than the original number of field equations, just as the number of variables $\hat{q}$ will be less than the number of components of a generic spacetime metric.

As with the field equations, the Lagrangian $\lambda(g)$ when restricted from $\mathcal{Q}$ to $\mathcal{Q}^{G}$ determines a (much simpler) reduced Lagrangian $\hat{\lambda}(\hat{q})$ for the fields $\hat{q}$. The construction of the reduced Lagrangian is slightly more complicated than the reduced field equations because the original Lagrangian is an $n$-form on $M$ and the reduced Lagrangian must be an $(n-l)$-form on $M / G$, where $l$ is the dimension of the group orbits in $M$. Let us therefore spell out the definition of the reduced Lagrangian in some detail.

We define a $G$-invariant $l$-chain $\chi$ on $M$ to be a totally antisymmetric tensor of type $\left(\begin{array}{l}l \\ 0\end{array}\right)$ which (1) is a $G$-invariant tensor field:

$$
\mu_{\gamma}^{*} \chi=\chi, \quad \forall \gamma \in G
$$

* See Appendix B for an explanation of how we are defining differential equations to be "equivalent". 
and (2) is everywhere tangent to the orbits of $G$ on $M$. This last requirement means that at any point $x \in M$ we have

$$
\chi=\chi^{i_{1} \cdots i_{l}} X_{i_{1}} \cdots X_{i_{l}}
$$

where $\left(X_{i_{1}}, X_{i_{2}}, \ldots, X_{i_{l}}\right)$ are a linearly independent set of generators of the isometry group action (Killing vector fields) at $x$. Suppose that $\omega=\omega(g)$ is a $G$-equivariant $p$-form locally constructed from the metric and its derivatives:

$$
\omega\left(\mu_{\gamma}^{*} g\right)=\mu_{\gamma}^{*} \omega(g), \quad \forall \gamma \in G
$$

Consider the $(p-l)$-form $\chi-\omega(g(\hat{q}))$ obtained from $\omega$ by contraction with $\chi$ and evaluation on a $G$-invariant metric $g(\hat{q}) \in \mathcal{Q}^{G}$. This form is $G$-invariant and satisfies

$$
X-\{\chi-\omega(g(\hat{q}))\}=0
$$

with $X$ being any infinitesimal generator of the group action $\mu$ on $M{ }^{*}$ This means that there exists a unique $(p-l)$-form $\hat{\omega}(\hat{q})$ on $M / G$ locally constructed from the fields $\hat{q}$ and their derivatives such that

$$
\chi-\omega(g(\hat{q}))=\pi^{*} \hat{\omega}(\hat{q}),
$$

where $\pi: M \rightarrow M / G$ is the projection from $M$ to its quotient by the $G$ action. So, given a choice of $G$-invariant $l$-chain on $M$, we have a map,

$$
\rho_{\chi}:\left(\Omega^{p}(M)\right)^{G} \rightarrow \Omega^{p-l}(M / G),
$$

that associates a $(p-l)$ form $\hat{\omega}(\hat{q})$ on $M / G$, to every $G$-invariant spacetime $p$-form $\omega(g(\hat{q}))$ :

$$
\rho_{\chi}(\omega(g(\hat{q})))=\hat{\omega}(\hat{q})
$$

We call $\rho_{\chi}$ the reduction map associated to the $l$-chain $\chi$.

If we restrict the Lagrangian to $\mathcal{Q}^{G}$, the resulting form $\lambda(g(\hat{q}))$ is $G$-invariant. We can now apply the reduction map to obtain the reduced Lagrangian $\hat{\lambda}(\hat{q})$ :

$$
\hat{\lambda}(\hat{q})=\rho_{\chi}(\lambda(g(\hat{q})))
$$

Associated to this reduced Lagrangian is a system of Euler-Lagrange equations $E(\hat{\lambda})=0$, for the fields $\hat{q}$ on $M / G$, where $E(\hat{\lambda})$ is defined via the first variational formula

$$
\delta \hat{\lambda}=E(\hat{\lambda}) \cdot \delta \hat{q}+d \hat{\eta}(\delta \hat{q})
$$

with $\delta \hat{q} \in \hat{\mathcal{S}}$. We emphasize that the reduced Lagrangian and its Euler-Lagrange form depend upon the choice made for the invariant chain $\chi$.

* $\overline{\text { Such forms are called basic. }}$ 
The construction of the reduction map and the reduced Lagrangian goes through when $\chi$ is only defined on a $G$-invariant open set $U \subset M$. In this case, the reduction map defines forms, e.g., the reduced Lagrangian $\hat{\lambda}$, only on $V=\pi(U) \subset M / G$. All of our preceding and subsequent considerations will apply in this setting provided we replace $M$ by $U$ and $M / G$ by $V=U / G$. And, as usual, we remind the reader that the foregoing discussion admits a purely local, infinitesimal description. One simply replaces the group $G$ acting on $M$ with an algebra of vector fields $\Gamma$ on an open set $U \subset M$ and uses the corresponding notions of infinitesimal invariance/equivariance. In this setting, the reduced Lagrangian and field equations are obtained by restricting to $\mathcal{S}^{\Gamma}$ and proceeding as before.

\section{The Principle of Symmetric Criticality}

The principle of symmetric criticality can now be stated in terms of the ingredients described in the previous sections.

Definition 5.1. A group action obeys the Principle of Symmetric Criticality (PSC) if about each $x \in M$ there exists a $G$-invariant open neighborhood $U$ and a $G$-invariant chain on $U$ such that, for any $G$-equivariant Lagrangian $\lambda$, the reduced field equations are equivalent (in the sense of Appendix B) to the Euler-Lagrange equations of the reduced Lagrangian $\hat{\lambda}$,

$$
E(\lambda)(g(\hat{q}))=0 \Longleftrightarrow E(\hat{\lambda})(\hat{q})=0
$$

We emphasize that PSC is a property of a group action and not a property of a specific Lagrangian. It is possible to have a particular Lagrangian that yields a correct reduced Lagrangian for some symmetry reduction even if PSC fails in the sense that not all Lagrangians can be successfully reduced. As an extreme example, the Lagrangian $\lambda=0$ will always reduce to give the correct field equations even if PSC is not valid for the chosen group action. The point of PSC, when it is valid, is that it guarantees the reduced Lagrangian is correct irrespective of the starting Lagrangian.

PSC guarantees that solutions to $E(\hat{\lambda})=0$ are in one-to-one correspondence with $G$-invariant solutions to $E(\lambda)=0 . \dagger$ This happens, for example, with the spherically symmetric reductions mentioned in $\S 1$. However, as pointed out in $\S 1$, PSC need not be valid. As we shall see, there are exactly two obstructions to the validity of PSC in relativistic field theory and they can be characterized quite explicitly in terms of properties of the symmetry group action $(G, \mu)$.

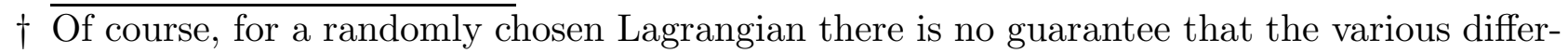
ential equations admit any solutions at all! The notion of equivalence we have adopted for defining PSC (see Appendix B) is particularly convenient in this regard since it only relies upon the relation between differential equations, not on any properties of their solution spaces - not to mention that such properties may be difficult to establish. 
In order to obtain conditions for the validity of PSC, we want to compare the EulerLagrange form $E(\lambda)(g(\hat{q}))$ with the Euler-Lagrange form of the reduced Lagrangian, $E(\hat{\lambda})(\hat{q})$. To this end, we note that the reduction map $\rho_{\chi}$, since it is defined independently of the metric, commutes with the process of field variation so that

$$
\begin{aligned}
\delta \hat{\lambda} & =\rho_{\chi}\left(\delta_{\hat{q}} \lambda(g(\hat{q}))\right) \\
& =\rho_{\chi}\left(E(\lambda)(g(\hat{q})) \cdot \delta_{\hat{q}} g(\hat{q})+d \eta\left(\delta_{\hat{q}} g(\hat{q})\right)\right) .
\end{aligned}
$$

In (5.2) we have introduced the notation $\delta_{\hat{q}}$, which indicates a variation in the fields $\hat{q}$ on $M / G$. In particular,

$$
\delta_{\hat{q}} g=P \cdot \delta \hat{q},
$$

where $P$ is the zeroth order linear differential operator defined in (2.6). Thus, $P$ is playing the role of the differential of the map $\Phi$ in (2.5) and $\delta_{\hat{q}} g$ represents a tangent vector at $g(\hat{q})$ to $\mathcal{Q}^{G}$.

As noted in $\S 4, \lambda, E(\lambda)$ and $\eta$ are $G$-equivariant. This means that $E(\lambda)(g(\hat{q})) \cdot \delta_{\hat{q}} g(\hat{q})$ and $d \eta\left(\delta_{\hat{q}} g(\hat{q})\right)$ are $G$-invariant forms on $M$. We can therefore apply $\rho_{\chi}$ to each term on the right-hand side of (5.2). Comparing (5.2) with (4.3) we have

$$
E(\hat{\lambda}) \cdot \delta \hat{q}+d \hat{\eta}(\delta \hat{q})=\rho_{\chi}(E(\lambda)(g(\hat{q})) \cdot(P \cdot \delta \hat{q}))+\rho_{\chi}(d \eta(P \cdot \delta \hat{q})) .
$$

Comparing this with (5.1), we have the following conditions for the validity of PSC.

Theorem 5.2. The following two conditions are necessary and sufficient for the validity of PSC.

PSC1: About each $x \in M$ there exists a $G$-invariant open neighborhood $U$ and a $G$ invariant chain $\chi$ on $U$ such that for each $G$-invariant $(n-1)$-form $\eta$ on $U$ there exists an $(n-l-1)$-form $\hat{\eta}$ on $U / G$ with

$$
\rho_{\chi}(d \eta)=d \hat{\eta} .
$$

Here $l$ is the dimension of the group orbits in $M$.

PSC2: For all G-equivariant Lagrangians $\lambda$

$$
E(\lambda)(g(\hat{q})) \cdot P=0 \quad \Longleftrightarrow \quad E(\lambda)(g(\hat{q}))=0
$$

Proof: These conditions can be seen to be sufficient for PSC as follows. Eq. (5.5) in PSC1 implies that

$$
\rho_{\chi}(d \eta(P \cdot \delta \hat{q}))=d \hat{\eta}(\delta \hat{q})
$$


in (5.4). Therefore we have that $E(\hat{\lambda})$ is determined by the "volume term" on the righthand side of (5.4):

$$
E(\hat{\lambda}) \cdot \delta \hat{q}=\rho_{\chi}(E(\lambda)(g(\hat{q})) \cdot(P \cdot \delta \hat{q})) \quad \forall \delta \hat{q}
$$

Since $\rho_{\chi}:\left(\Omega^{n}(U)\right)^{G} \rightarrow \Omega^{n-l}(U / G)$ is an isomorphism, it follows from (5.7) that the EulerLagrange equations of the reduced Lagrangian are always equivalent to at least a subset of the correct reduced field equations, namely,

$$
E(\hat{\lambda})=0 \Longleftrightarrow E(\lambda)(g(\hat{q})) \cdot P=0 .
$$

Equation (5.6) of PSC2 then reduces (5.8) to (5.1). Conditions PSC1 and PSC2 are necessary for PSC since PSC asserts that (5.1) holds for all G-equivariant Lagrangians. If PSC1 or PSC2 is not satisfied there will be some Lagrangian for which the reduced Lagrangian will not yield valid Euler-Lagrange equations. We will exhibit such PSCviolating Lagrangians to $§ 5.1$ and $§ 5.2$.

The role of PSC1 is to guarantee that the restriction of the boundary term to $\mathcal{Q}^{G}$ does not introduce any additional "volume terms" proportional to $\delta \hat{q}$ on the right hand side of (5.4). Such terms will have the effect of supplying erroneous contributions to the EulerLagrange equations of the reduced Lagrangian, rendering these equations incorrect. Thus PSC1 guarantees that the field equations produced by the reduced Lagrangian correctly yield at least a subset of the reduced field equations. We note that the Bianchi class B models mentioned in $\S 1$ do not satisfy PSC1, and this is the reason PSC fails in these models. Granted PSC1, the role of PSC2 is to guarantee that the reduced Lagrangian supplies all of the reduced field equations.

In the next two subsections we provide pointwise conditions on the group action which are necessary and sufficient for PSC1 and PSC2. In the third subsection a number of special situations are considered where the validity of these pointwise conditions can be established.

\subsection{PSC1: The Lie algebra condition}

PSC1 has already been studied in [12] under the hypotheses of (i) the existence of a $G$ equivariant boundary term $\eta$ in the variational principle (3.1), (ii) a transverse symmetry group action, and (iii) connected $G$ and $G_{x}$. As mentioned in $\S 4$, in general relativity we always have a $G$-equivariant boundary term. Moreover, it is straightforward to check that the analysis of PSC1 appearing in [12] does not, in fact, depend upon the transversality assumption and that these results immediately generalize to allow for a disconnected symmetry group and/or isotropy subgroups. So, referring to [12] for proofs where necessary, we have the following results. 
Proposition 5.3. $P S C 1$ is equivalent to the condition that about each point $x \in M$ there is a $G$-invariant open neighborhood $U$ and $G$-invariant chain $\chi$ on $U$ such that

$$
\rho_{\chi}(d \eta)=d \rho_{\chi}(\eta),
$$

for all $G$-invariant differential forms $\eta$ on $U$.

Proof: : See [12].

Therefore, following [12], if a $G$-invariant chain $\chi$ exists on a $G$-invariant open set $U$, so that PSC1 holds in $U$, we say that $\chi$ defines a cochain map $\rho_{\chi}:\left(\tilde{\Omega}^{*}(U)\right)^{G} \rightarrow \Omega^{*-l}(U / G)$, from the space of $G$-basic forms on $U$ to the corresponding forms on $U / G$. PSC1 requires that a cochain map exist in a $G$-invariant neighborhood of any point $x \in M$. Necessary and sufficient conditions for the existence of a cochain map in a $G$-invariant open set, expressed in terms of properties of the group action, are as follows.

Proposition 5.4. There exists a cochain map $\rho_{\chi}$ satisfying (5.9) on a $G$-invariant open set $U$ if and only if there exists on $U$ a $G$-invariant chain that has vanishing Lie derivative along all $G$-invariant vector fields on $U$.

Proof: : See [12].

The condition stated in Proposition 5.4 for the existence of a cochain map can be reformulated in terms of the relative Lie algebra cohomology $\mathcal{H}^{*}\left(\Gamma, G_{x}\right)$ of the Lie algebra $\Gamma$ of $G$ relative to its isotropy subgroups $G_{x}$. The cohomology $\mathcal{H}^{l}\left(\Gamma, G_{x}\right)$ at degree $l$ is defined as follows. Fix a basis $e_{a}, a=1,2, \ldots, \operatorname{dim} G$ for the Lie algebra. The structure constants of the Lie algebra are then defined by the Lie bracket:

$$
\left[e_{a}, e_{b}\right]=C_{a b}{ }^{c} e_{c} .
$$

The Lie algebra $\Gamma$ can be viewed as the space of left-invariant vector fields on the manifold $G$ and the Lie bracket as the vector field commutator. The dual basis of left-invariant 1-forms on $G, \omega^{a}, a=1,2, \ldots, \operatorname{dim} G$, satisfy

$$
d \omega^{a}=-\frac{1}{2} C_{b c}{ }^{a} \omega^{b} \wedge \omega^{c} .
$$

The Lie algebra cohomology of $G$ at degree $l$ is defined as the space of closed $l$-forms on $G$ modulo the exact $l$-forms, with all forms being left- $G$-invariant. It can be computed using the exterior derivative formula (5.10). The relative Lie algebra cohomology $\mathcal{H}^{l}\left(\Gamma, G_{x}\right)$ is defined as the set of closed $l$-forms on the group $G$ modulo the set of exact $l$ forms, where all forms are left- $G$-invariant and right $G_{x}$-basic. This last condition means that, with 
$\Gamma_{x}=\operatorname{Lie}\left(G_{x}\right)$, all forms are required to be invariant under the right action of $G_{x}$ on $G$ and to satisfy

$$
X\lrcorner \omega=0, \quad \forall X \in \Gamma_{x} .
$$

A $G_{x}$-basic form on $G$ is the pull-back of a form on $G / G_{x}$. The relative Lie algebra cohomology $\mathcal{H}^{*}\left(\Gamma, G_{x}\right)$ computes the $G$-invariant de Rham cohomology of the orbit $G / G_{x}$ through $x \in M$.

Let $l$ be the dimension of the group orbits in $M$. Then we have the following necessary and sufficient condition for PSC1.

Proposition 5.5. Around each point $x \in M$ there is a $G$-invariant neighborhood $U$ such that a cochain map exists on $U$ if and only if

$$
\mathcal{H}^{l}\left(\Gamma, G_{x}\right) \neq 0, \quad \forall x \in M .
$$

Proof: See [12] for a proof of existence of a cochain map in a neighborhood of each point $x \in M$ when (5.11) holds. It is straightforward to check that this neighborhood can always be chosen to be $G$-invariant.

We call (5.11) the Lie algebra condition for PSC. If $x$ and $x^{\prime}$ lie in the same $G$ orbit then $\mathcal{H}^{*}\left(\Gamma, G_{x}\right)$ is isomorphic to $\mathcal{H}^{*}\left(\Gamma, G_{x^{\prime}}\right)$, so one need only check the Lie algebra condition along a cross section of the group action.

Finally, we note that Proposition 5.4 can be used to show the necessity of PSC1 in Theorem 5.2. Suppose that PSC1 does not hold, then there is no cochain map and according to Proposition 5.4 there will exist a $G$-invariant vector field $S$ such that $L_{S} \chi \neq 0$ for any $G$-invariant chain $\chi$. In fact, as shown in [12], $S$ can be chosen tangent to the group orbits and there exists a smooth, non-trivial $f$ such that

$$
L_{S} \chi=f \chi,
$$

where both $S$ and $f$ can be chosen independently of the $G$-invariant $l$-chain $\chi$. Consider the trivial Lagrangian

$$
\begin{aligned}
\lambda(g) & =d(S\lrcorner \epsilon(g)) \\
& =L_{S} \epsilon(g) \\
& =\frac{1}{2} g^{\mu \nu}\left(L_{S} g_{\mu \nu}\right) \epsilon(g),
\end{aligned}
$$

where $\epsilon(g)$ is the volume form of $g$. Being exact, this Lagrangian has identically vanishing Euler-Lagrange form. On the other hand, if we compute the pull back of the reduced Lagrangian $\hat{\lambda}$ to $M$, we have

$$
\begin{aligned}
\pi^{*} \hat{\lambda}(\hat{q}) & =\chi\lrcorner \lambda(g(\hat{q})) \\
& \left.\left.=L_{S}(\chi\lrcorner \epsilon(g(\hat{q}))\right)-\left(L_{S} \chi\right)\right\lrcorner \epsilon(g(\hat{q})) .
\end{aligned}
$$


The first term in (5.14) is zero because $\chi-\epsilon(g(\hat{q}))$ is basic and of degree $(n-l)$. Therefore

$$
\pi^{*} \hat{\lambda}(\hat{q})=-f \chi-\epsilon(g(\hat{q}))
$$

which implies that the reduced Lagrangian is of order zero in $\hat{q}$ and is non-trivial provided the space of invariant metrics is non-trivial. Therefore $\hat{\lambda}$ has non-vanishing Euler-Lagrange form, which violates PSC.

\subsection{PSC2: The Palais condition}

For the derivation of the results of this section and their subsequent application to various examples in $\S 6$, it is convenient to define the symmetric $\left(\begin{array}{l}2 \\ 0\end{array}\right)$ tensor field $\Delta(g) \in \mathcal{S}^{*}$ by

$$
E(\lambda(g))=\Delta(g) \otimes \epsilon(g),
$$

where $\epsilon(g)$ is the volume form of the metric $g$. For example, in (3.6) where $\lambda(g)$ is the Einstein-Hilbert Lagrangian form, $\Delta(g)$ is minus the Einstein tensor. Note that $\Delta(g)$ is $G$-equivariant if $\lambda(g)$ is. This implies that $\Delta(g(\hat{q}))$ is a $G$-invariant symmetric $\left(\begin{array}{l}2 \\ 0\end{array}\right)$ tensor field, $\Delta(g(\hat{q})) \in\left(\mathcal{S}^{*}\right)^{G}$. From (5.15), PSC2 is equivalent to the statement that, for all field equations coming from $G$-equivariant Lagrangians,

$$
\Delta(g(\hat{q})) \cdot P=0 \Longleftrightarrow \Delta(g(\hat{q}))=0 .
$$

To describe necessary and sufficient conditions for (5.16) to be satisfied, we recall some definitions from $\S 2$. Let $S_{x}=T_{x}^{*} M \odot T_{x}^{*} M$ be the set of possible values of symmetric $\left(\begin{array}{l}0 \\ 2\end{array}\right)$ tensor fields at the point $x \in M$ and denote by $S_{x}^{G_{x}} \subset S_{x}$ the vector space of $G_{x}$-invariant symmetric $\left(\begin{array}{l}0 \\ 2\end{array}\right)$ tensors at $x \in M$. At each $x \in M, \delta_{\hat{q}} g(\hat{q})_{x} \in S_{x}^{G x}$. Similarly, we have the dual representation of the group $G_{x}$ acting on $S_{x}^{*}=T_{x} M \odot T_{x} M$ and we denote the fixed points of this action as $\left(S_{x}^{*}\right)^{G_{x}}$, so that $\left(S_{x}^{*}\right)^{G_{x}} \subset S_{x}^{*}$ is the vector space of $G_{x}$-invariant $\left(\begin{array}{l}2 \\ 0\end{array}\right)$ tensors at $x \in M$. By equivariance of $\Delta$, at each point $x \in M$ we have that

$$
\Delta(g(\hat{q}))_{x} \in\left(S_{x}^{*}\right)^{G_{x}} .
$$

Finally, let us denote by $\left(S_{x}^{G_{x}}\right)^{0} \subset S_{x}^{*}$ the annihilator of $S_{x}^{G x}$. $\left(S_{x}^{G_{x}}\right)^{0}$ is the set of elements $\omega \in S_{x}^{*}$ such that $\omega \cdot h=0$ for all $h \in S_{x}^{G x}$.

Proposition 5.6. A necessary and sufficient condition for the validity of (5.16) - and hence PSC2 - is that

$$
\left(S_{x}^{*}\right)^{G_{x}} \cap\left(S_{x}^{G_{x}}\right)^{0}=0, \quad \forall x \in M
$$

Proof: Recall that $\hat{\mathcal{S}}$ is the space of sections of a vector bundle over $M / G$ that parametrizes $\mathcal{S}^{G}$. Let us denote by $\hat{\mathcal{S}}^{*}$ the space of sections of the dual vector bundle. Define the linear mapping

$$
H:\left(\mathcal{S}^{*}\right)^{G} \rightarrow \hat{\mathcal{S}}^{*}
$$


by

$$
H(v)=v \cdot P, \quad v \in\left(\mathcal{S}^{*}\right)^{G},
$$

so that

$$
\Delta(g(\hat{q})) \cdot P=H(\Delta(g(\hat{q})))
$$

Because

$$
P: \hat{\mathcal{S}} \rightarrow \mathcal{S}^{G}
$$

is an isomorphism, it follows that $H$ is surjective. Therefore to prove sufficiency of (5.18) for PSC2 (in the form (5.16)) it is enough to show that $H$ is injective when (5.18) holds. We have that

$$
\operatorname{ker}(H)=\left\{v \in\left(\mathcal{S}^{*}\right)^{G} \mid v \cdot w=0, \forall w \in \mathcal{S}^{G}\right\} .
$$

Now, if $v \in\left(\mathcal{S}^{*}\right)^{G}$ then at each $x \in M$ we have that $v_{x} \in\left(S_{x}^{*}\right)^{G_{x}}$. Our regularity assumptions on the group action guarantee that for each element $w_{x} \in S_{x}^{G x}$ there is a smooth section $w \in \mathcal{S}^{G}$ taking the value $w_{x}$ at $x \in M$. Therefore, if $v \in \operatorname{ker}(H)$ then $v_{x} \in\left(S_{x}^{*}\right)^{G_{x}} \cap\left(S_{x}^{G x}\right)^{0}$ so that condition (5.18) forces $\operatorname{ker}(H)=0$, i.e., (5.18) implies that $H$ is an isomorphism and PSC2 holds.

To verify necessity of (5.18) for PSC2, fix $h_{x} \in\left(S_{x}^{*}\right)^{G_{x}} \cap\left(S_{x}^{G_{x}}\right)^{0}$ and let $h \in \mathcal{S}^{*}$ be any $G$-invariant symmetric $\left(\begin{array}{l}2 \\ 0\end{array}\right)$ tensor field on $M$ that takes the value $h_{x}$ at $x$. Such a form exists by virtue of the regularity assumptions we have made on the group action $[13,16]$. Let $\omega$ be a $G$-invariant volume form on $M$. Such a form always exists since we assume the existence of a $G$-invariant metric. Consider the $G$-equivariant Lagrangian

$$
\lambda=h^{\alpha \beta} g_{\alpha \beta} \omega .
$$

PSC2 requires that

$$
h \cdot P=0, \quad \Longleftrightarrow \quad h=0 .
$$

At the point $x,(5.20)$ implies that $h_{x}=0$. Therefore, (5.18) is necessary and sufficient for PSC2 to hold.

In the context of PSC for $G$-invariant functionals on Banach manifolds Palais has arrived at condition (5.18) [11], but with $G$ acting on $\mathcal{S}$ rather than $G_{x}$ acting on $S_{x}$ as we have here. We therefore refer to (5.18) as the Palais condition for PSC. Note, however, that unlike the case in [11], condition (5.18) is necessary but not sufficient for PSC since (5.18) only guarantees PSC2. It is clear that the Palais condition can only fail when (1) the group action is not free, i.e., there is non-trivial isotropy, and (2) the isotropy group is represented non-trivially on the space of values of the fields at each point. Conditions (1) and (2) imply the action of the symmetry group on the bundle of fields is necessarily nontransverse. So, for transverse group actions (such as considered in [12]), PSC2 is always satisfied. 
Finally, we note that equation (5.19) is an example of a $G$-equivariant Lagrangian that violates PSC when PSC2 does not hold, thus establishing the necessity of PSC2 in Theorem 5.2.

\subsection{Further developments, simplifications and specializations}

Here we provide some additional results on the Lie algebra condition (5.11) and the Palais condition (5.18) for PSC, given that the group actions of interest preserve a spacetime metric and/or granted additional simplifying assumptions. We begin by characterizing the Palais condition in terms of inner products on $S_{x}^{G x}$ and then show that the Palais condition is always satisfied if there is a $G$-invariant Riemannian metric on $M$. We then assume that $G$ and $G_{x}$ are connected so that the Lie algebra and Palais conditions for PSC can be characterized using infinitesimal methods.

Proposition 5.7. Condition (5.18) holds if and only if every $G_{x}$ invariant metric on $S_{x}$ is non-degenerate when restricted to $S_{x}^{G_{x}}$.

Proof: Let $B: S_{x} \times S_{x} \rightarrow \mathbb{R}$ be a $G_{x}$-invariant, non-degenerate quadratic form on the space of symmetric $\left(\begin{array}{l}0 \\ 2\end{array}\right)$ tensors at $x \in M$. This means that if $h$ and $k$ are any symmetric $\left(\begin{array}{l}0 \\ 2\end{array}\right)$ tensors we have

$$
B\left(\mu_{\gamma}^{*} h, \mu_{\gamma}^{*} k\right)=B(h, k), \quad \forall \gamma \in G_{x} .
$$

Because we are assuming the group action leaves a metric invariant, such quadratic forms always exist. For example, if $g \in \mathcal{Q}^{G}$, then we can set

$$
B(h, k)=g^{\alpha \beta} g^{\gamma \delta} h_{\alpha \gamma} k_{\beta \delta}
$$

Given $h \in S_{x}^{G x}$ we can use $B$ to define $\alpha \in\left(S_{x}^{*}\right)^{G_{x}}$ by "raising indices" in the usual way. Thus $B$ defines an isomorphism:

$$
B: S_{x}^{G_{x}} \rightarrow\left(S_{x}^{*}\right)^{G_{x}}
$$

Likewise, it is straightforward to see that, in addition, $B$ defines an isomorphism:

$$
B: S_{x}^{0} \rightarrow\left(\mathcal{S}_{x}^{G x}\right)^{\perp}
$$

where $\left(\mathcal{S}_{x}^{G_{x}}\right)^{\perp}$ is the orthogonal complement to $S_{x}^{G_{x}}$ in $S_{x}$ with respect to $B$. We thus get an isomorphism

$$
B:\left(S_{x}^{*}\right)^{G_{x}} \cap S_{x}^{0} \rightarrow S_{x}^{G x} \cap\left(\mathcal{S}_{x}^{G x}\right)^{\perp},
$$

and the Palais condition is equivalent to

$$
S_{x}^{G_{x}} \cap\left(\mathcal{S}_{x}^{G_{x}}\right)^{\perp}=0, \quad \forall x \in M
$$


for all $G_{x}$-invariant metrics on the space $S_{x}$. Evidently, the Palais condition fails if and only if $S_{x}^{G x}$ and its orthogonal complement with respect to every $G_{x}$-invariant metric on $S_{x}$ have common elements. This occurs precisely when the restriction of $B$ to $S_{x}^{G_{x}}$ is degenerate.

Corollary 5.8. Condition (5.18) is satisfied if there there exists at least one positive definite $G_{x}$-invariant scalar product on $T_{x} M$.

Proof: Using the positive definite scalar product in $(5.21)$ the resulting metric on $S_{x}$ is positive-definite and will not become degenerate on any subspace. From Lemma 5.7, (5.18) must be satisfied.

Corollary 5.9. Condition (5.18) is satisfied if there exists a G-invariant Riemannian metric on $M$.

Proof: At each point $x \in M$ a $G$-invariant Riemannian metric defines a positive definite $G_{x}$-invariant scalar product on $T_{x} M$.

This last result shows that PSC2 holds when considering symmetry reduction of a theory involving a Riemannian metric, that is, in "Euclidean gravity" theories.

Corollary 5.10. Condition (5.18) is satisfied for group actions with compact isotropy groups.

Proof: A compact isotropy group will admit a $G_{x}$-invariant positive-definite quadratic form at each $x$. Once again this leads to a positive definite metric (5.21).

Note, however, that a compact isotropy group need not prevent the failure PSC1. Indeed, this condition can fail when the group action is free and the group is not unimodular (see below).

From the preceding considerations we obtain a very useful sufficient condition for the validity of PSC.

Proposition 5.11. PSC is valid when $G$ is a compact Lie group.

Proof: As noted in [12] when $G$ is compact the Lie algebra condition (5.11) is satisfied. And, because the isotropy subgroups will be compact, the Palais condition is satisfied by Corollary 5.10.

This result has been established by Palais [11] for PSC defined in terms of $G$-invariant functions on Banach manifolds. Note, in particular, that Proposition 5.11 shows that PSC is valid when considering reductions according to spherical symmetry. 
For the rest of this section we specialize to the common situation where the isotropy subgroups $G_{x}$ are connected so that infinitesimal methods can be used to characterize the two conditions for PSC. We begin with some definitions. At a given point $x \in M$, let $\mathbf{h} \subset \Gamma$ be the Lie algebra of the isotropy group $G_{x}$. Define $\mathbf{m}$ to be the vector space

$$
\mathbf{m}=\Gamma / \mathbf{h} .
$$

The tangent space to the $G$-orbit at $x$ can be identified with $\mathbf{m}$ and the infinitesimal linear isotropy representation on the tangent space to the orbit at $x$ is then identified with the adjoint representation $a d_{\mathbf{h}}: \mathbf{h} \rightarrow g l(\mathbf{m})$ of $\mathbf{h}$ on $\mathbf{m}:$

$$
\operatorname{ad}_{\mathbf{h}} v=[v, \mathbf{h}] \bmod \mathbf{h}, \quad v \in \mathbf{m} .
$$

The normalizer $\mathbf{n}(\mathbf{h})$ is the largest subalgebra of $\Gamma$ that contains $\mathbf{h}$ as an ideal:

$$
[\mathbf{n}(\mathbf{h}), \mathbf{h}] \subset \mathbf{h} .
$$

Let $a d_{\mathbf{n}}: \mathbf{n} \rightarrow g l(\mathbf{m})$ be the adjoint representation of $\mathbf{n}$ on $\mathbf{m}$ given by

$$
a d_{\mathbf{n}} v=[v, \mathbf{n}] \bmod \mathbf{h}, \quad v \in \mathbf{m}
$$

We define the Lie algebra $\mathbf{s} \subset \mathbf{m}$ by

$$
\mathbf{s}=\mathbf{n}(\mathbf{h}) / \mathbf{h} .
$$

It is not hard to see that the isotropy subalgebra $\mathbf{h}$ acts trivially on $\mathbf{s}$. The space $\mathbf{s}$ corresponds to the values at $x$ of $G$-invariant vector fields tangent to the orbit through $x$. We denote by $a d_{\mathbf{s}}: \mathbf{s} \rightarrow g l(\mathbf{m})$ the restriction of $a d_{\mathbf{n}}$ to $\mathbf{s}$.

Proposition 5.12. For connected $G_{x}$ with Lie algebra $\mathbf{h}$ and normalizer $\mathbf{n}(\mathbf{h})$, (5.11) is equivalent to

$$
\operatorname{tr}\left(a d_{\mathbf{n}}\right)=0
$$

or equivalently,

$$
\begin{aligned}
& \operatorname{tr}\left(a d_{\mathbf{h}}\right)=0, \\
& \operatorname{tr}\left(a d_{\mathbf{s}}\right)=0,
\end{aligned}
$$

for all $x \in M$.

Proof: Pick a complement for $\mathbf{h} \subset \Gamma$ and identify it with $\mathbf{m}$. Let us choose a basis $\tau_{i}$ for $\mathbf{h}$ and $\tau_{\alpha}$ for $\mathbf{m}$. In terms of structure constants, condition (5.22) is equivalent to the two conditions: 
(i)

$$
C_{i \alpha}{ }^{\alpha}=0
$$

(ii) If $v^{\alpha} \in \mathbf{m}$ satisfies

$$
v^{\alpha} C_{i \alpha}^{\beta}=0,
$$

then it also satisfies

$$
v^{\alpha} C_{\alpha \beta}^{\beta}=0
$$

Eq. (5.25) is equivalent to (5.23) and eq. (5.26)-(5.27) is equivalent to (5.24). As shown in $[12]$, conditions (i) and (ii) are equivalent to (5.11).

We remark that the conditions (5.22), (5.23) and (5.24) mean that the adjoint actions of $\mathbf{n}, \mathbf{h}$ and $\mathbf{s}$ on $\mathbf{m}$ are each the infinitesimal action of a unimodular group. In addition, $v \in \mathbf{s}$ if and only if it satisfies (5.26), and then $v$ represents the value of a $G$-invariant vector field $V$ tangent to the orbit at $x$. Condition (5.27) is equivalent to the statement that the vector field $V$ is divergence-free (at $x$ ) relative to any $G$-invariant volume element on the orbit (cf. Proposition 5.4).

Proposition 5.13. If $G_{x}$ is connected and $\mathbf{m}$ admits a non-degenerate bilinear form invariant under the action of $\mathbf{h},(5.11)$ is equivalent to

$$
\operatorname{tr}\left(a d_{\mathbf{s}}\right)=0
$$

at all $x \in M$.

Proof: As in Proposition 5.12, pick a complement for $\mathbf{h} \subset \Gamma$ and identify it with $\mathbf{m}$. Denote by $\tau_{i}$ a basis for $\mathbf{h}$ and denote by $\tau_{\alpha}$ a basis for $\mathbf{m}$. Let $\sigma_{\alpha \beta}$ be the components of the quadratic form $\sigma: \mathbf{m} \times \mathbf{m} \rightarrow \mathbb{R}$ in the chosen basis for $\mathbf{m}$. By (infinitesimal) $G_{x}$ invariance, these components satisfy

$$
C_{i \alpha}^{\gamma} \sigma_{\gamma \beta}+C_{i \beta}{ }^{\gamma} \sigma_{\alpha \gamma}=0, \quad \forall i
$$

Contraction with $\sigma^{\alpha \beta}$ implies that (5.23) (equivalently, (5.25)) is satisfied in Proposition 5.12 .

If there exists a $G$-invariant metric on spacetime that induces a non-degenerate metric on the orbits, so that the orbits are either spacelike or timelike in the Lorentzian case, then this defines a non-degenerate bilinear form on $\mathbf{m}$. We therefore have the following result.

Corollary 5.14. For a group action with $G_{x}$ connected such that there exists a $G$ invariant metric with respect to which the group orbits are non-null, the Lie algebra condition (5.11) is satisfied if and only if

$$
\operatorname{tr}\left(a d_{\mathbf{s}}\right)=0
$$


for all $x \in M$.

We remark that the Lie algebra condition (5.11) will be satisfied according to Proposition 5.13 or 5.14 whenever $\mathbf{s}$ is trivial or its representation on $\mathbf{m}$ is trivial. The former case occurs when there are no $G$-invariant vector fields tangent to the orbits so that $\mathbf{s}=0$. The latter case occurs when the $G$-invariant vector fields tangent to the orbits correspond to elements of the center $\mathbf{c}$ of the Lie algebra, i.e., $\mathbf{n}(\mathbf{h})=\mathbf{h}+\mathbf{k}$, where $\mathbf{k} \subset \mathbf{c}$. These two situations occur frequently among the vector field algebras listed in [15].

Let us briefly consider the special case where the group action is free, that is, the isotropy group at each point is trivial. This case is already treated in [12] (see also [8]); here it becomes a corollary to Proposition 5.12. We define $a d_{\Gamma}$ to be the infinitesimal adjoint representation of the Lie algebra $\Gamma$ on itself.

Corollary 5.15. For a free, connected group action PSC is equivalent to the unimodular condition

$$
\operatorname{tr}\left(\operatorname{ad}_{\Gamma}\right)=0
$$

Proof: For a free action $\mathbf{h}=0$ and $\mathbf{m}=\mathbf{s}=\mathbf{n}=\Gamma$. The condition (5.22) becomes simply (5.30). Because the group action is free, the Palais condition (5.18) for PSC is satisfied. Thus (5.30) is necessary and sufficient for PSC when the group $G$ is connected and acting freely.

When the symmetry group is acting freely with spacelike hypersurface orbits then we are considering spatially homogeneous cosmological models. In a four-dimensional spacetime these models are often called the "Bianchi models" since they are determined by a choice of connected three-dimensional Lie group, all of which have been classified by Bianchi. The condition (5.30), which is equivalent to the structure constant condition

$$
C_{i j}{ }^{j}=0,
$$

picks out the Bianchi class A cosmological models as obeying PSC $[2,4,8]$.

Let us conclude this section by giving a simple necessary and sufficient condition for the Palais condition under the hypothesis that the isotropy group of any given point is connected.

Proposition 5.16. Let $G$ act by isometries on a four dimensional Lorentz manifold with $G_{x}$ connected for all $x \in M$. A necessary and sufficient condition for (5.18) is that the linear isotropy representation at any $x \in M$ not correspond to one of the the null rotation subgroups.

Proof: Since at each point $x \in M$ the linear isotropy representation of $G_{x}$ must preserve a quadratic form of Lorentz signature, it follows that the linear isotropy representation 
defines a conjugacy class of a subgroup of the Lorentz group. Granted that $G_{x}$ is connected, this conjugacy class is completely characterized by its Lie algebra. Up to conjugation, there are 14 distinct subalgebras of the Lorentz Lie algebra ranging in dimensions from 1 to 6 [21]. It is straightforward to compute the intersection appearing in (5.18) for each of the 14 subalgebras of the Lorentz algebra. We find that the subalgebras generating null rotation subgroups are the only subalgebras that correspond to infinitesimal linear isotropy representations which fail to satisfy (5.18). A null rotation subgroup of the Lorentz group has the property that it leaves invariant one and only one null vector $N$. The tensor $N \otimes N$ defines a non-vanishing element of $\left(S_{x}^{*}\right)^{G_{x}} \cap\left(S_{x}^{G_{x}}\right)^{0}$ (there may be other non-vanishing elements).

There are 3 null rotation conjugacy classes of $S O(3,1)$ with dimensions 1,2 , and 3 . Given an isometry group, there is a straightforward, coordinate/frame independent way of testing whether the linear isotropy representation at a point corresponds to one of the null rotation subgroups thus violating PSC. First, at a given point $x \in M$, one computes the infinitesimal linear isotropy representation as a Lie algebra of linear transformations of $T_{x} M$. We assume that the linear isotropy representation is of dimension 1,2 or 3 , otherwise (5.18) is satisfied. If the dimension is 1 or 2 , then the linear isotropy representation corresponds to a null rotation if and only if each element of the infinitesimal linear isotropy algebra has vanishing eigenvalues. If the dimension of $G_{x}$ is 3 , then $G_{x}$ corresponds to a null rotation if the infinitesimal linear isotropy algebra admits an element with 2 non-zero imaginary eigenvalues and an element admitting only zero eigenvalues.

\subsection{Summary of key results on PSC}

Let us summarize the salient results of this section. Let a group $G$ act on $M$ with orbits of fixed dimension $l$ and such that the regularity assumptions given in $[13,16]$ are satisfied. Denote the Lie algebra of $G$ by $\Gamma$ and the isotropy group of $x \in M$ by $G_{x}$. Denote by $S_{x}^{G_{x}}$ the vector space of $G_{x}$-invariant symmetric $\left(\begin{array}{l}0 \\ 2\end{array}\right)$ tensors at $x$, denote by $\left(S_{x}^{*}\right)^{G_{x}}$ the

vector space of $G_{x}$-invariant symmetric $\left(\begin{array}{l}2 \\ 0\end{array}\right)$ tensors at $x$, and denote by $\left(S_{x}^{G_{x}}\right)^{0} \subset S_{x}^{*}$ the annihilator of $S_{x}^{G_{x}}$. Let $\mathcal{H}^{l}\left(\Gamma, G_{x}\right)$ be the Lie algebra cohomology at degree $l$ of $\Gamma$ relative to $G_{x} \subset G$.

Theorem 5.17. The following two conditions are necessary and sufficient for PSC:

(i) $\mathcal{H}^{l}\left(\Gamma, G_{x}\right) \neq 0, \forall x \in M \quad$ (Lie algebra condition)

(ii) $\left(S_{x}^{*}\right)^{G_{x}} \cap\left(S_{x}^{G x}\right)^{0}=0, \forall x \in M . \quad$ (Palais condition)

In addition,

- if $G$ is compact then PSC is valid; 
- if the group action is free then PSC is valid if and only if $G$ is unimodular;

- if there exists a $G$-invariant Riemannian metric on $M$, then PSC is valid if and only if the Lie algebra condition holds;

- in four dimensions, if the metrics under consideration are Lorentzian and $G_{x}$ is connected, PSC is satisfied if and only if the Lie algebra condition holds and $G_{x}$ is not equivalent to a null rotation subgroup of the Lorentz group, $\forall x \in M$.

All these results on PSC have local, infinitesimal versions, which are obtained by replacing the group $G$ acting on $M$ with a Lie algebra of vector fields $\Gamma$ on an open set $U \subset M$. In particular, in Theorem 5.17 we replace $G_{x}$ with $\Gamma_{x}$ in order to get necessary and sufficient conditions for PSC in the infinitesimal setting. Of course, when $\Gamma$ and $\Gamma_{x}$ are the infinitesimal generators of a connected $G$ action with connected isotropy $G_{x}$ at each $x$, then the validity of PSC using $\Gamma$ and $\Gamma_{x}$ is equivalent to the validity of PSC using $G$ and $G_{x}$.

\section{Examples}

We begin by briefly considering examples in which the isotropy groups are zerodimensional, i.e., trivial or discrete. We next examine in some detail various examples involving four-dimensional group actions with three dimensional orbits. These examples are particularly nice since (i) the three dimensional orbits force the reduced field equations to be ODEs, and (ii) the one-dimensional isotropy group at each point reduces the number of arbitrary functions in the metric to 4 , so that the various formulas for the field equations, Lagrangians, and Euler-Lagrange equations are manageable. Finally we consider a couple of transitive group actions. One novel feature of this latter class of examples is that the field equations and Euler-Lagrange equations are always algebraic in the free parameters that characterize the group-invariant metrics.

In each of the following examples we define the (local) symmetry group action, either explicitly or infinitesimally, and we check the validity of PSC according to the results of $\S 5$. We also compute the invariant metrics, the reduced Einstein equations, the reduced Einstein-Hilbert Lagrangian, and the Euler-Lagrange equations of the reduced Lagrangian so as to illustrate the validity or failure of PSC. In each of the examples the invariant metrics on $M$ can be expressed as

$$
g(q)=q^{i} h_{i},
$$

where the $q^{i}: M \rightarrow \mathbb{R}^{m}$ are $G$ - invariant functions on $M$ and the $h_{i}$ are a basis for $\mathcal{S}^{G}$. Since $G$ invariant functions on $M$ are in 1-1 correspondence with functions on $M / G$, we can identify the functions $q^{i}$ with the fields $\hat{q}$. This identification $\hat{q} \leftrightarrow q$ allows us to 
perform computations on the reduced spacetime manifold by using invariant functions on $M$. In particular, the field equations, when restricted to the $G$-invariant metrics on $M$, can be expressed as

$$
E(\lambda)(g(q))=\Delta(g(q)) \otimes \epsilon=0
$$

with

$$
\Delta(g(q))=\Delta_{i}(q) f^{i}
$$

where $f^{i}$ are a basis for $\left(\mathcal{S}^{*}\right)^{G}$. The functions $\Delta_{i}(q)$ are a set of $G$ invariant differential operators on $M$. The reduced equations $\hat{\Delta}(\hat{q})=0$ are identified with the equations $\Delta_{i}(q)=$ 0 . Likewise, the reduced Lagrangian is identified with the invariant form

$$
\hat{\lambda}(q)=\chi-\lambda(g(q))
$$

on $M$, from which we can compute representatives of the Euler-Lagrange equations of the reduced Lagrangian using the identification $E(\hat{\lambda}(\hat{q}))=0 \Longleftrightarrow E(\hat{\lambda}(q))=0$.

We remind the reader that all of these considerations apply in the infinitesimal setting in which $G$ is replaced with $\Gamma, G_{x}$ is replaced with $\Gamma_{x}$, and so forth.

\subsection{Freely acting Abelian groups.}

Free Abelian group actions are found in the "one Killing vector models" and the "two Killing vector models", etc. For simplicity we will focus on the case of a two dimensional group. Let us suppose that $M=\mathbb{R}^{4}$ (although other topologies are possible) with coordinates $x^{\alpha}, \alpha=1, \ldots, 4$. We consider the translation group action whose infinitesimal generators are

$$
X_{1}=\partial_{1}, \quad X_{2}=\partial_{2}
$$

Points in the reduced spacetime manifold $M / G \approx \mathbb{R}^{2}$ can be labeled by $\left(x^{3}, x^{4}\right)$. Every $G$-invariant metric can be expressed as

$$
g(q)=q_{\alpha \beta}\left(x^{3}, x^{4}\right) d x^{\alpha} \otimes d x^{\beta}, \quad q_{\alpha \beta}=q_{\beta \alpha} .
$$

Thus the space of $G$-invariant metrics can be viewed as the space of fields $q_{\alpha \beta}$ on $M / G$.

Freely acting groups trivially satisfy PSC2. Moreover, for such group actions PSC1 is satisfied since the group is unimodular (see Corollary 5.15). Indeed, for free Abelian group actions (1) all left-invariant forms on $G$ are right- $G_{x}$-basic since $G_{x}$ is trivial, and (2) all left $G$-invariant forms on the group are closed - so there cannot be any exact forms, and every form represents a cohomology class. Thus we have

$$
\mathcal{H}^{2}(\Gamma, I d)=\mathbb{R}
$$


and a cochain map always exists according to Proposition 5.5. Up to a multiplicative constant, it is induced by the $G$-invariant chain

$$
\chi=\partial_{1} \wedge \partial_{2}
$$

Using the fact that all $G$-invariant vector fields are of the form $v^{\alpha} \partial_{\alpha}$ with $v^{\alpha}=v^{\alpha}\left(x^{3}, x^{4}\right)$, it is easy to check that $\chi$ in (6.2) satisfies Proposition 5.4 and defines a cochain map via (4.2).

The reduced Einstein equations are rather well studied in this context (see, for example, [22]), and can be obtained by simply dropping all derivatives with respect to $x^{1}$ and $x^{2}$ in the field equations. Likewise, the reduced Lagrangian $\hat{\lambda}$ can be obtained by simply dropping all derivatives with respect to $x^{1}$ and $x^{2}$ in the Einstein-Hilbert Lagrangian and then contracting with the chain (6.2). It is a standard exercise to see that the EulerLagrange equations of $\hat{\lambda}$ must agree with the reduced field equations. Of course, this result is also guaranteed by our general theory, i.e., the fact that $\chi$ defines a cochain map.

\subsection{Orthogonally transitive group actions}

We next consider the special case of orthogonally transitive two dimensional Abelian isometry groups. Recall that a group action is orthogonally transitive if, for any $G$-invariant metric, the distribution $\mathcal{D} \subset T M$ orthogonal to the group orbits is integrable. This definition is slightly awkward since it uses the infinite dimensional space of $G$-invariant metrics to characterize the isometry group action. As mentioned in $\S 2$, we prefer to view the group action as simply a Lie group $G$ of diffeomorphisms of a manifold $M$, which then is used to select the allowed $G$-invariant metrics. For our purposes, a superior but equivalent - definition of an orthogonally transitive group action is as the semi-direct product of a discrete $\left(Z_{2}\right)$ group, with action

$$
\left(x^{1}, x^{2}\right) \rightarrow\left(-\epsilon x^{1},-\epsilon x^{2}\right), \quad \epsilon= \pm 1
$$

and the $\left(x^{1}, x^{2}\right)$ translation group, which is a normal subgroup. The non-trivial action of the isotropy group $G_{x_{0}}$ of a point with coordinates $x_{0}^{\alpha}$ is given by

$$
x^{\alpha} \rightarrow\left(-x^{1}+2 x_{0}^{1},-x^{2}+2 x_{0}^{2}, x^{3}, x^{4}\right)
$$

The reduced spacetime manifold $M / G$ can still be parametrized by $\left(x^{3}, x^{4}\right)$. Because of the isotropy constraint (2.4) the $G$-invariant metrics are now of the form

$$
g(q)=q_{a b}\left(x^{i}\right) d x^{a} \otimes d x^{b}+\tilde{q}_{i j}\left(x^{i}\right) d x^{i} \otimes d x^{j}, \quad q_{a b}=q_{b a}, q_{i j}=q_{j i} \quad a, b=1,2 \quad i, j=3,4,
$$


so that $\mathcal{Q}^{G}$ is the set of fields parametrized by $q_{a b}$ and $\tilde{q}_{i j}$ on $M / G$, constrained so that $g(q)$ has Lorentz signature.

From (6.4) it is clear that, with respect to any $G$-invariant metric, the orbit-orthogonal distribution $\mathcal{D}$ is integrable. Conversely, according to the traditional definition, every metric admitting an orthogonally transitive group action can be put into the form (6.4), so that the group action and $G$-invariant metrics we have described are equivalent to the usual notion of an orthogonally transitive group action.

Let us now consider the two conditions for PSC. Of course, it is well known that the Einstein-Hilbert Lagrangian does reduce to give a valid Lagrangian for the $G$-invariant spacetimes (see, e.g., [23]). We shall see that, in fact, there are no obstructions to PSC, thus guaranteeing the successful reduction of any ( $G$-invariant) Lagrangian.

The Lie algebra condition for PSC can be computed as follows. Fix a basis $\left(e_{1}, e_{2}\right)$ for the Abelian Lie algebra $\Gamma$ of the isometry group. The dual basis of left- $G$-invariant forms on $G$ are denoted $\left(\omega^{1}, \omega^{2}\right)$ and satisfy

$$
d \omega^{1}=0=d \omega^{2} .
$$

The right-action of $G_{x}$ on the dual basis is given by

$$
\omega^{i} \longrightarrow-\omega^{i}, \quad i=1,2 .
$$

The closed, right- $G_{x}$-basic 2 -forms on $G$ are proportional to

$$
\omega=\omega^{1} \wedge \omega^{2} .
$$

Since all left- $G$-invariant forms are closed, $\omega$ cannot be exact and we see that $\mathcal{H}^{2}\left(\Gamma, G_{x}\right)=\mathbb{R}$ for all $x \in M$. Equivalently, it is easy to see that the chain from the previous example,

$$
\chi=\partial_{1} \wedge \partial_{2},
$$

is $G$-invariant and still defines a cochain map.

To check the Palais condition (5.18) for PSC we compute

$$
\begin{gathered}
S_{x}^{G x}=\left\{d x^{a} \odot d x^{b}, d x^{i} \odot d x^{j}\right\}, \quad a, b=1,2 \quad i, j=3,4, \\
\left(S_{x}^{*}\right)^{G x}=\left\{\partial_{a} \odot \partial_{b}, \partial_{i} \odot \partial_{j}\right\}, \quad a, b=1,2 \quad i, j=3,4,
\end{gathered}
$$

and

$$
\left(S_{x}^{G_{x}}\right)^{0}=\left\{\partial_{a} \odot \partial_{i}\right\}
$$

so that

$$
\left(S_{x}^{*}\right)^{G_{x}} \cap\left(S_{x}^{G_{x}}\right)^{0}=0 .
$$


(Here we introduce the notation in which $\{W\}$ is the vector space spanned by the vectors $W)$.

These computations show that PSC is satisfied for the orthogonally transitive 2 Killing vector models, irrespective of the choice of Lagrangian for the metric. This is, of course, easy to verify directly. For example, consider the vacuum Einstein theory described by the Einstein-Hilbert Lagrangian. The reduced Lagrangian is obtained as in the free group case considered previously by (1) dropping all derivatives with respect to $x^{1}$ and $x^{2},(2)$ setting $g_{a i}=0,(3)$ contracting with the chain $\chi$. It then follows that the Euler-Lagrange equations of the reduced Lagrangian correspond to the field equations

$$
\mathcal{G}_{a b}=0=\mathcal{G}_{i j}
$$

for the metrics of the form (6.4). It is easy to check that for the metrics (6.4) the reduced field equations are equivalent to (6.5). In particular, the missing components $\mathcal{G}_{a i}$ are identically zero for metrics (6.4) admitting the orthogonally transitive group action. This follows from the $G$-invariance of the Einstein tensor when evaluated on a $G$-invariant metric.

\subsection{Stationary, spherically symmetric spacetimes}

Here we consider the well-known case of a spacetime admitting time translational and rotational symmetry. Of course, for reductions by the group $S O(3)$ PSC is guaranteed thanks to the compactness of $S O(3)$ (see Proposition 5.11). But the addition of time translation symmetry renders the symmetry group non-compact, so a closer look is warranted.

The spacetime manifold can be taken to be $M=\mathbb{R}^{+} \times \mathbb{R} \times S^{2}$. The abstract Lie group is $G=\mathbb{R} \times S O(3)$ with orbits $\mathbb{R} \times S^{2}$. The isotropy group $G_{x}$ of any point is isomorphic to $S O(2) \subset S O(3)$. The reduced spacetime is $M / G \approx \mathbb{R}^{+}$. Since $G$ and $G_{x}$ are connected, we will use infinitesimal methods to study PSC.

The $G$-invariant metrics are

$$
g(q)=q^{1}(r) d t \otimes d t+q^{2}(r) d t \odot d r+q^{3}(r) d r \otimes d r+\frac{1}{2} q^{4}(r) d \Omega^{2}=q^{i} h_{i},
$$

where $r$ labels the group orbits, $d t$ and $d r$ are $G$-invariant 1-forms, $d \Omega^{2}$ is the standard metric on $S^{2}, q^{i}=q^{i}(r), i=1,2,3,4$ with

$$
\left(q^{4}\right)^{2}\left(\left(q^{2}\right)^{2}-4 q^{1} q^{3}\right)>0
$$

are the dependent variables for the reduced theory, and the $h_{i}$ are a basis for the $G$-invariant symmetric $\left(\begin{array}{l}0 \\ 2\end{array}\right)$ tensor fields. 
About any given point in $M$ we will use a spherical polar coordinate chart $(t, r, \theta, \phi)$, where

$$
d \Omega^{2}=\left(d \theta \otimes d \theta+\sin ^{2} \theta d \phi \otimes d \phi\right) .
$$

Points in $M / G$ will be labeled by $r$. A set of infinitesimal generators of the group action are expressed in the coordinate chart as

$$
X_{1}=\frac{\partial}{\partial \phi}, \quad X_{2}=\sin \phi \frac{\partial}{\partial \theta}+\cos \phi \cot \theta \frac{\partial}{\partial \phi}, \quad X_{3}=\cos \phi \frac{\partial}{\partial \theta}-\sin \phi \cot \theta \frac{\partial}{\partial \phi}, \quad X_{4}=\frac{\partial}{\partial t} .
$$

This algebra of vector fields $\Gamma$ spanned by $\left(X_{1}, \ldots, X_{4}\right)$ appears in Petrov's classification [15] as (32.9). At a generic point, $x_{0}^{\alpha}=\left(t_{0}, r_{0}, \theta_{0}, \phi_{0}\right)$ the infinitesimal generator of $G_{x_{0}}$ is given in coordinates by

$$
Y=-\cot \theta_{0} X_{1}+\cos \phi_{0} X_{2}-\sin \phi_{0} X_{3}
$$

The infinitesimal linear isotropy representation at $x_{0}$ is then given in this chart by

$$
\left.Y_{, \beta}^{\alpha}\right|_{x_{0}}=\left(\begin{array}{cccc}
0 & 0 & 0 & 0 \\
0 & 0 & 0 & 0 \\
0 & 0 & 0 & 1 \\
0 & 0 & -\csc ^{2} \theta_{0} & 0
\end{array}\right)
$$

Let us first consider PSC2. The eigenvalues of the matrix (6.7) constitute a complex conjugate, pure imaginary pair corresponding to the fact that $G_{x}=S O(2)$. We conclude, by Proposition 5.16, that the Palais condition - and hence PSC2 - is satisfied.

We next consider PSC1. The Lie group $G$ and its isotropy subgroups are connected and the orbits of the group action are reductive homogeneous spaces $\frac{\mathbb{R} \times S O(3)}{S O(2)}$. Let us view $\Gamma$ as an abstract Lie algebra with basis $X_{i}, i=1,2, \ldots, 4$. At the point $x_{0}$, defined by $t=$ const, $r=$ const., $\theta=\frac{\pi}{2}, \phi=\frac{\pi}{2}$, we have the reductive decomposition $\Gamma=\mathbf{h}+\mathbf{m}$, where

$$
\mathbf{h}=\left\{X_{3}\right\}, \quad \mathbf{m}=\left\{X_{1}, X_{2}, X_{4}\right\}
$$

It is easy to check that, at any point,

$$
\mathbf{n}(\mathbf{h}) \cap \mathbf{m}=\left\{X_{4}\right\}
$$

and that $X_{4}$ spans the center of $\Gamma$. Moreover, the $G$-invariant metrics include the Minkowski metric, which induces a non-degenerate metric on the group orbits. According to Corollary 5.14 the Lie algebra condition - and hence PSC1 - is satisfied.

It is straightforward to compute $\mathcal{H}^{3}\left(\Gamma, G_{x}\right)$ directly. Denote by $\omega^{i}, i=1,2,3,4$ the basis of left-invariant differential forms on $G$ dual to the basis $X_{i}$. This dual basis satisfies

$$
d \omega^{i}=-\frac{1}{2} \epsilon_{j k}^{i} \omega^{j} \wedge \omega^{k}, \quad i, j, k=1,2,3,
$$


and

$$
d \omega^{4}=0
$$

At the point $x_{0}$ defined above, the right- $G_{x_{0}}$-basic closed 3 -forms are proportional to $\omega^{1} \wedge \omega^{2} \wedge \omega^{4}$ while the right- $G_{x_{0}}$-basic 2 -forms are all proportional to $\omega^{1} \wedge \omega^{2}$. Since

$$
d\left(\omega^{1} \wedge \omega^{2}\right)=0
$$

it follows that $\omega^{1} \wedge \omega^{2} \wedge \omega^{4}$ cannot be exact, and hence $\mathcal{H}^{3}\left(\Gamma, G_{x_{0}}\right)=\mathbb{R}$. It is not hard to see that this result is valid for any $x \in M$.

From our general theory we know that a suitable cochain map exists. In a spherical polar coordinate chart it is given by

$$
\chi=\frac{1}{\sin \theta} \frac{\partial}{\partial t} \wedge \frac{\partial}{\partial \theta} \wedge \frac{\partial}{\partial \phi} .
$$

$\chi$ is $G$-invariant and satisfies

$$
L_{\frac{\partial}{\partial t}} \chi=0=L_{\frac{\partial}{\partial r}} \chi
$$

Note that any invariant vector field is a combination of $\left(\partial_{t}, \partial_{r}\right)$ with $r$-dependent coefficients, so that a cochain map exists according to Proposition 5.4.

Let us consider the symmetry reduction of the vacuum Einstein equations $\mathcal{G}(g)=0$. The restriction of the Einstein tensor to a $G$-invariant metric $g(q)$ is given by

$$
\mathcal{G}(g(q))=\mathcal{G}_{i}(q) f^{i}
$$

where $\mathcal{G}_{i}$ are second order differential functions of the $q^{i}$, and the $f^{i}$ are a basis of $G$ invariant symmetric $\left(\begin{array}{l}2 \\ 0\end{array}\right)$ tensors dual to the $h_{i}$. In the spherical polar coordinate chart they are:

$$
f^{i}=\left(\frac{\partial}{\partial t} \otimes \frac{\partial}{\partial t}, \frac{\partial}{\partial t} \odot \frac{\partial}{\partial r}, \frac{\partial}{\partial r} \otimes \frac{\partial}{\partial r}, \frac{\partial}{\partial \theta} \otimes \frac{\partial}{\partial \theta}+\frac{1}{\sin ^{2} \theta} \frac{\partial}{\partial \phi} \otimes \frac{\partial}{\partial \phi}\right) .
$$

With some purely algebraic rearrangements, the 4 field equations $\mathcal{G}_{i}=0$ are equivalent to 3 algebraically independent equations

$$
\begin{gathered}
q^{1 \prime}=-\frac{q^{1}\left(q^{4 \prime}\right)^{2}+2 q^{4}}{2 q^{4} q^{4 \prime}} \\
q^{1 \prime \prime}=\frac{q^{1}\left(q^{4 \prime}\right)^{2}+2 q^{4}}{2\left(q^{4}\right)^{2}} \\
q^{4 \prime \prime}=\frac{\left(q^{4 \prime}\right)^{2}}{2 q^{4}}
\end{gathered}
$$


where

$$
F^{\prime}:=\frac{1}{\sqrt{\left(q^{2}\right)^{2}-4 q^{1} q^{3}}} \frac{d}{d r} F .
$$

The fact that only 3 field equations are algebraically independent is a consequence of the contracted Bianchi identities, which provide an algebraic relationship among the four field equations that are allowed by $G$-invariance.

Let us now consider the reduction of the Einstein-Hilbert Lagrangian,

$$
\lambda=R(g) \epsilon(g)
$$

Restricting this Lagrangian to the $G$-invariant metric $g(q)$ in (6.6) yields

$$
\lambda(g(q))=\frac{1}{2 q^{4}} \sqrt{\left(q^{2}\right)^{2}-4 q^{1} q^{3}}\left(4 q^{1} q^{4} q^{4 \prime \prime}-q^{1}\left(q^{4 \prime}\right)^{2}+2\left(q^{4}\right)^{2} q^{1 \prime \prime}+4 q^{4} q^{4 \prime} q^{1 \prime}+2 q^{4}\right) \nu,
$$

where, in spherical polar coordinates,

$$
\nu=\sin \theta d t \wedge d r \wedge d \theta \wedge d \phi
$$

Using (6.8), and using the radius $r$ as a coordinate on $M / G$, the reduced Lagrangian is given by

$$
\begin{aligned}
\hat{\lambda}(q) & =\chi-(\lambda(g(q))) \\
& =\frac{1}{2 q^{4}} \sqrt{\left(q^{2}\right)^{2}-4 q^{1} q^{3}}\left(4 q^{1} q^{4} q^{4 \prime \prime}-q^{1}\left(q^{4 \prime}\right)^{2}+2\left(q^{4}\right)^{2} q^{1 \prime \prime}+4 q^{4} q^{4 \prime} q^{1 \prime}+2 q^{4}\right) d r .
\end{aligned}
$$

A straightforward, if a bit lengthy, computation reveals that the 4 Euler-Lagrange equations of $\hat{\lambda}(q)$ are equivalent to the equations $\mathcal{G}_{i}=0$, or (6.10)-(6.13). In fact, denoting by $E_{i}$ the Euler-Lagrange expressions obtained for each of the $q^{i}$, we have that

$$
E_{i}(\hat{\lambda}(q))=-q^{4} \sqrt{\left(q^{2}\right)^{2}-q^{1} q^{3}} \mathcal{G}_{i} .
$$

\subsection{Locally isotropic Bianchi class B}

This example can be viewed as a special case of the Bianchi class B cosmological models mentioned in the introduction. In local coordinates $\left(\lambda^{1}, \lambda^{2}, \lambda^{3}\right) \in \mathbb{R}^{3}, \lambda^{4} \in(0,2 \pi)$ on $G$ and in coordinates $x^{\alpha}, \alpha=1, \ldots, 4$ on $M=\mathbb{R}^{4}$ we define a four dimensional group action

$$
x^{\alpha} \longrightarrow \mu^{\alpha}(\lambda, x)
$$

by

$\mu^{\alpha}(\lambda, x)=\left(x^{1}-\lambda^{3}, \lambda^{1}+e^{\lambda^{3}}\left[x^{2} \cos \left(\lambda^{4}\right)-x^{3} \sin \left(\lambda^{4}\right)\right], \lambda^{2}+e^{\lambda^{3}}\left[x^{3} \cos \left(\lambda^{4}\right)+x^{2} \sin \left(\lambda^{4}\right)\right], x^{4}\right)$. 
The reduced spacetime $M / G \approx \mathbb{R}$ can be parametrized by $x^{4}$. The isotropy groups $G_{x}$ are all connected and isomorphic to $S O(2)$. For example, at the origin $x_{0}^{\alpha}=(0,0,0,0)$ the action of $G_{x_{0}}$ is given in local coordinates by

$$
\mu^{\alpha}(\lambda, x)=\left(x^{1}, x^{2} \cos \left(\lambda^{4}\right)-x^{3} \sin \left(\lambda^{4}\right), x^{3} \cos \left(\lambda^{4}\right)+x^{2} \sin \left(\lambda^{4}\right), x^{4}\right) .
$$

The group action (6.14) has a Lie algebra $\Gamma$ of infinitesimal generators spanned by

$$
X_{1}=\frac{\partial}{\partial x^{2}}, \quad X_{2}=\frac{\partial}{\partial x^{3}}, \quad X_{3}=-\frac{\partial}{\partial x^{1}}+x^{2} \frac{\partial}{\partial x^{2}}+x^{3} \frac{\partial}{\partial x^{3}}, \quad X_{4}=x^{2} \frac{\partial}{\partial x^{3}}-x^{3} \frac{\partial}{\partial x^{2}},
$$

which corresponds to (32.6) in [15]. The generator of the isotropy subgroup of the origin, (6.15), is $X_{4}$.

The general form of a $G$-invariant metric is

$g(q)=\frac{1}{2} q^{1} e^{2 x^{1}}\left[d x^{2} \otimes d x^{2}+d x^{3} \otimes d x^{3}\right]+q^{2} d x^{1} \otimes d x^{1}-q^{3} d x^{1} \odot d x^{4}+q^{4} d x^{4} \otimes d x^{4}=q^{i} h_{i}$,

where $q^{i}=q^{i}\left(x^{4}\right), i=1, \ldots, 4$ are arbitrary except for the signature requirement

$$
\left(q^{1}\right)^{2}\left[4 q^{2} q^{4}-\left(q^{3}\right)^{2}\right]<0 .
$$

We first consider the Lie algebra condition for PSC. Because $G$ and $G_{x}$ are connected, we can use Proposition 5.12 to check (5.11). Let us view $\Gamma$ as an abstract Lie algebra with basis $X_{i}, i=1,2, \ldots, 4$. At $x_{0}^{\alpha}=(0,0,0,0)$ the isotropy subalgebra is generated by $X_{4}$ and we have the reductive decomposition $\Gamma=\mathbf{h}+\mathbf{m}$, with $\mathbf{h}=\left\{X_{4}\right\}$ and $\mathbf{m}=\left\{X_{1}, X_{2}, X_{3}\right\}$. In addition, we have that (at $\left.x_{0}\right) \mathbf{n}(\mathbf{h}) \cap \mathbf{m}=\left\{X_{3}\right\}$. Since

$$
\sum_{j=1}^{3} C_{3}^{j}=-2,
$$

we see that (5.24) is not satisfied. It is not hard to see that a similar result occurs at each point in $M$, and so PSC1 fails.

Let us check the failure of PSC1 by explicitly computing $\mathcal{H}^{3}\left(\Gamma, G_{x_{0}}\right)$. Using the basis $\omega^{i}$ of left-invariant 1-forms on $G$ dual to the $X_{i}$, it is not hard to check that all $G_{x_{0}}$ basic closed 3-forms on $G$ are proportional to

$$
\alpha=\omega^{1} \wedge \omega^{2} \wedge \omega^{3} .
$$

It is likewise straightforward to check that

$$
\beta=\omega^{2} \wedge \omega^{1}
$$


is a $G_{x_{0}}$ basic 2 -form and that

$$
\alpha=d \beta,
$$

so that

$$
\mathcal{H}^{3}\left(\Gamma, G_{x_{0}}\right)=0,
$$

confirming the failure of PSC1.

The preceding computations indicate that no suitable cochain map will exist. Indeed, it is easy to check that any $G$-invariant chain is a function of $x^{4}$ multiplied by

$$
\chi=e^{-2 x^{1}} \frac{\partial}{\partial x^{1}} \wedge \frac{\partial}{\partial x^{2}} \wedge \frac{\partial}{\partial x^{3}} .
$$

Any $G$-invariant vector field tangent to an orbit is a function of $x^{4}$ multiplied by

$$
S=\frac{\partial}{\partial x^{1}} .
$$

A $G$-invariant chain cannot be invariant under the flow generated by a $G$-invariant vector field tangent to the orbits since

$$
L_{S} \chi=-2 \chi .
$$

Thus, by Proposition 5.4 there can be no cochain map, i.e., PSC1 fails.

As for PSC2, it is straightforward to check that at a generic point $x_{0}^{\alpha}$ the isotropy algebra $\Gamma_{x_{0}}$ is generated by

$$
Y=x_{0}^{3} X_{1}-x_{0}^{2} X_{2}+X_{4},
$$

so that the infinitesimal linear isotropy representation is given by

$$
\left.Y_{, \beta}^{\alpha}\right|_{x_{0}}=\left(\begin{array}{cccc}
0 & 0 & 0 & 0 \\
0 & 0 & -1 & 0 \\
0 & 1 & 0 & 0 \\
0 & 0 & 0 & 0
\end{array}\right),
$$

which has pure imaginary, complex conjugate eigenvalues. As in the previous example, the linear isotropy representation is that of a spatial rotation, $S O(2)$, and the Palais condition for PSC is satisfied.

The restriction of the Einstein tensor $\mathcal{G}$ to a $G$-invariant metric is given by

$$
\mathcal{G}(g(q)):=\mathcal{G}_{i}(q) f^{i},
$$

where $\mathcal{G}_{i}$ are second order differential functions of the $q^{i}$, and the $f^{i}$ are a basis for $\left(\mathcal{S}^{*}\right)^{G}$. With some purely algebraic rearrangements, the equations $\mathcal{G}_{i}=0$ are equivalent to

$$
\left(D q^{1}\right)^{2}-\frac{1}{\left(q^{2}\right)^{2}}=0,
$$




$$
\begin{gathered}
\left(q^{2 \prime}\right)^{2}-1=0, \\
\left(D q^{1}\right)^{\prime}+\frac{1}{\left(q^{2}\right)^{2}}=0, \\
q^{2 \prime \prime}=0
\end{gathered}
$$

where

$$
D q^{1}=\frac{1}{\sqrt{\left(q^{3}\right)^{2}-4 q^{2} q^{4}}}\left(\frac{1}{q^{1}} \frac{d q^{1}}{d x^{4}}+\frac{q^{3}}{q^{2}}\right)
$$

and the prime denotes

$$
F^{\prime}:=\frac{1}{\sqrt{\left(q^{3}\right)^{2}-4 q^{2} q^{4}}} \frac{d F}{d x^{4}} .
$$

Restricting the Einstein-Hilbert Lagrangian to a $G$-invariant metric gives

$$
\begin{aligned}
\lambda(g(q))=\frac{e^{2 x^{1}}}{2 q^{2}} \sqrt{\left(q^{1}\right)^{2}\left[\left(q^{3}\right)^{2}-4 q^{2} q^{4}\right]}[ & -3+2 q^{2} q^{2 \prime \prime}+3\left(D q^{1}\right)^{2}\left(q^{2}\right)^{2} \\
& \left.+4\left(D q^{1}\right)^{\prime}\left(q^{2}\right)^{2}+4 q^{2} D q^{1} q^{2 \prime}\right] d x^{1} \wedge d x^{2} \wedge d x^{3} \wedge d x^{4}
\end{aligned}
$$

To construct a reduced Lagrangian we need to pick a $G$-invariant chain $\chi$. Since $\chi$ can't be further specified by demanding it define a cochain map there is some arbitrariness in our choice of $\chi$. In particular, we can always multiply $\chi$ by any function of $x^{4}$ and keep the chain $G$-invariant. The most general reduced Lagrangian is thus obtained by using (6.18) multiplied by a function $f\left(x^{4}\right)$. We then have

$\hat{\lambda}=\frac{f\left(x^{4}\right)}{2 q^{2}} \sqrt{\left(q^{1}\right)^{2}\left[\left(q^{3}\right)^{2}-4 q^{2} q^{4}\right]}\left(2 q^{2} q^{2 \prime \prime}+3\left(q^{2}\right)^{2}\left(D q^{1}\right)^{2}+4\left(q^{2}\right)^{2}\left(D q^{1}\right)^{\prime}+4 q^{2} q^{2 \prime} D q^{1}-3\right) d x^{4}$

There is no choice of function $f$ such that the Euler-Lagrange equations for $\hat{\lambda}$ are equivalent to the reduced field equations (6.19)-(6.22). For example, direct computation shows that the Euler-Lagrange equations of $\hat{\lambda}$ imply

$$
D q^{1}=\frac{2}{q^{2} \sqrt{\left(q^{3}\right)^{2}-4 q^{2} q^{4}}}\left(\frac{q^{2}}{f} \frac{d f}{d x^{4}}-q^{3}\right)
$$

which is clearly inequivalent to (6.19) for any choice of $f$.

\subsection{Null rotation isotropy}

Let us now examine an example for which PSC2 fails. Our example is taken from [15] (32.8). On $\mathbb{R}^{4}$, introduce coordinates $x^{\alpha}=\left(t, x^{1}, x^{2}, x^{3}\right)$ and a Lie algebra $\Gamma$ of vector fields spanned by

$$
X_{1}=e^{-x^{3}}\left(\frac{\partial}{\partial x^{1}}-\left(x^{2}\right)^{2} \frac{\partial}{\partial x^{2}}-2 x^{2} \frac{\partial}{\partial x^{3}}\right), \quad X_{2}=\frac{\partial}{\partial x^{3}}, \quad X_{3}=e^{x^{3}} \frac{\partial}{\partial x^{2}}, \quad X_{4}=\frac{\partial}{\partial x^{1}} .
$$


The isotropy subalgebra $\Gamma_{x_{0}}$ of a point $x_{0}^{\alpha}=\left(t_{0}, x_{0}^{1}, x_{0}^{2}, x_{0}^{3}\right)$ is generated by the vector field

$$
Y_{x_{0}}=e^{x_{0}^{3}} X_{1}+2 x_{0}^{2} X_{2}+e^{-x_{0}^{3}}\left(x_{0}^{2}\right)^{2} X_{3}-X_{4} \text {. }
$$

Note that the vector fields (6.23) only generate a local group action on $\mathbb{R}^{4}$ so we work with the infinitesimal version of PSC.

The general form of a $\Gamma$-invariant metric is

$$
\begin{aligned}
g(q)= & 4 q^{1} d x^{1} \odot d x^{2}-4 q^{1} x^{2} d x^{1} \odot d x^{3}+4 q^{2} d x^{2} \otimes d x^{2}-8 q^{2} x^{2} d x^{2} \odot d x^{3}+2 q^{3} d t \odot d x^{2} \\
& +\left(4 q^{2}\left(x^{2}\right)^{2}-q^{1}\right) d x^{3} \otimes d x^{3}-2 q^{3} x^{2} d t \odot d x^{3}+q^{4} d t \otimes d t \\
= & q^{i} h_{i}
\end{aligned}
$$

where $q^{i}=q^{i}(t), i=1, \ldots, 4$ are arbitrary except for the signature requirement

$$
q^{1} q^{4}<0
$$

We shall restrict attention to the case where

$$
q^{4}>0
$$

which implies that the group orbits are timelike.

PSC1 is satisfied in this example. At the origin the isotropy $\Gamma_{x_{0}}$ is generated by the vector field

$$
Y=X_{1}-X_{4}
$$

We view $\Gamma$ as an abstract Lie algebra with basis $e_{i}, i=1,2,3,4$, taken from (6.23) and with dual basis $\omega^{i}$. The space of $\Gamma_{x_{0}}$-basic closed 3 -forms on $\Gamma$ is spanned by the form

$$
\alpha=\omega^{1} \wedge \omega^{2} \wedge \omega^{3}-\omega^{2} \wedge \omega^{3} \wedge \omega^{4} .
$$

The space of $\Gamma_{x_{0}}$-basic 2 -forms on $\Gamma$ is spanned by the form

$$
\beta=\omega^{2} \wedge \omega^{3}
$$

Since $d \beta=0$, it follows that $H^{3}\left(\Gamma, \Gamma_{x_{0}}\right)=\mathbb{R}$. It is easy to see that this same result holds for all $x \in M$. A $\Gamma$-invariant chain that provides a cochain map is given by constant multiples of

$$
\chi=\frac{\partial}{\partial x^{1}} \wedge \frac{\partial}{\partial x^{2}} \wedge \frac{\partial}{\partial x^{3}}
$$

Since the module of $\Gamma$-invariant vector fields is generated by $\left(\frac{\partial}{\partial t}, \frac{\partial}{\partial x^{1}}\right)$ and since

$$
L_{\frac{\partial}{\partial t}} \chi=0=L_{\frac{\partial}{\partial x^{1}}} \chi
$$


it follows from Proposition 5.4 that $\chi$ defines the desired cochain map.

To see that PSC2 fails in this example, we compute the infinitesimal linear isotropy representation at a generic point $x_{0}^{\alpha}=\left(t_{0}, x_{0}^{1}, x_{0}^{2}, x_{0}^{3}\right)$. We find

$$
\left.Y_{, \beta}^{\alpha}\right|_{x_{0}}=\left(\begin{array}{cccc}
0 & 0 & -1 & 0 \\
0 & -2 x_{0}^{2} & 2\left(x_{0}^{2}\right)^{2} & 0 \\
0 & -2 & 2 x_{0}^{2} & 0 \\
0 & 0 & 0 & 0
\end{array}\right)
$$

The eigenvalues of (6.27) all vanish, so the infinitesimal linear isotropy representation corresponds to the Lorentz subalgebra of null rotations. Thus by Theorem 5.17 the Palais condition fails.

The restriction of the Einstein tensor $\mathcal{G}$ to a $\Gamma$-invariant metric $(6.25)$ is given by

$$
\mathcal{G}(g(q))=\mathcal{G}_{i}(q) f^{i}
$$

where $\mathcal{G}_{i}$ are second order differential functions of the $q^{i}$ and the $f^{i}$ are $\Gamma$-invariant tensor fields given by

$$
\begin{aligned}
& f^{i}=\left(\frac{\partial}{\partial x^{1}} \odot \frac{\partial}{\partial x^{2}}-\left(x^{2}\right)^{2} \frac{\partial}{\partial x^{2}} \otimes \frac{\partial}{\partial x^{2}}-2 x^{2} \frac{\partial}{\partial x^{2}} \odot \frac{\partial}{\partial x^{3}}-\frac{\partial}{\partial x^{3}} \otimes \frac{\partial}{\partial x^{3}},\right. \\
& \left.\frac{\partial}{\partial x^{1}} \otimes \frac{\partial}{\partial x^{1}}, \frac{\partial}{\partial x^{1}} \odot \frac{\partial}{\partial t}, \frac{\partial}{\partial t} \otimes \frac{\partial}{\partial t}\right) .
\end{aligned}
$$

With some purely algebraic rearrangements, the equations $\mathcal{G}_{i}=0$ are equivalent to the three equations

$$
\begin{gathered}
\left(q^{1 \prime}\right)^{2}-q^{1}=0, \\
q^{1 \prime \prime}-\frac{1}{2}=0, \\
q^{2 \prime \prime}=\frac{4 q^{2}+q^{1 \prime} q^{2 \prime}}{q^{1}}
\end{gathered}
$$

where the prime is defined by

$$
F^{\prime}=\frac{1}{\sqrt{q^{4}}} \frac{d F}{d t} .
$$

There are only three independent Einstein equations because the Bianchi identities provide an algebraic identity satisfied by the four field equations allowed by $G$-invariance.

The restriction of the Einstein-Hilbert Lagrangian to the $\Gamma$-invariant metric (6.25) is

$$
\lambda(g(q))=-3 \sqrt{-q^{1} q^{4}}\left(2 q^{1 \prime \prime}-1\right) d x^{1} \wedge d x^{2} \wedge d x^{3} \wedge d t .
$$

To construct a reduced Lagrangian we use the chain $\chi$ given in (6.26). This yields the reduced Lagrangian

$$
\hat{\lambda}=-3 \sqrt{-q^{1} q^{4}}\left(2 q^{1 \prime \prime}-1\right) d t
$$


Note that only two of the four dependent variables appear in the Lagrangian; there are only two non-trivial Euler-Lagrange equations, and they are equivalent to (6.29) and (6.30). The third field equation (6.31) does not follow from the reduced Lagrangian (6.32). That there is a single missing equation can be understood as follows. First, we note that $\left(S_{x}^{*}\right)^{\Gamma x}=\left\{f^{i}\right\}_{x}$, so that using (6.28) we have

$$
\left(S_{x}^{*}\right)^{\Gamma x} \cap\left(S_{x}^{\Gamma x}\right)^{0}=\left\{\frac{\partial}{\partial x^{1}} \otimes \frac{\partial}{\partial x^{1}}, \frac{\partial}{\partial x^{1}} \odot \frac{\partial}{\partial t}\right\}_{x}
$$

showing again that the Palais condition fails. Since (6.33) is two dimensional, there are two field equations which will not follow from the reduced Lagrangian. As noted earlier, the Bianchi identities imply that one of the field equations is not algebraically independent of the other three. This redundant equation happens to be one of the equations not provided by the reduced Lagrangian. So, at the end of the day, the reduced Lagrangian fails to provide only one of the independent field equations. Note that the Bianchi identities stem from full diffeomorphism equivariance - as opposed to $\Gamma$-equivariance - of the EinsteinHilbert Lagrangian, so the fact that only one rather than two field equations are missing is a consequence of a special choice of Lagrangian.

\subsection{Homogeneous spacetimes}

Homogeneous spacetimes (admitting a transitive isometry group) provide examples where the reduced field equations are purely algebraic. In addition, such symmetry reductions provide examples in which PSC is valid, in which PSC1 and PSC2 each fails separately, and in which they fail simultaneously. Given our previous examples, we will keep the presentation brief.

\section{Example 6.6a}

Let us begin with an example, taken from [15] eq. (33.23), in which PSC is valid. On $M=\mathbb{R}^{4}$ with coordinates $x^{\alpha}, \alpha=1, \ldots, 4$, the Lie algebra of vector fields $\Gamma$ is spanned by:

$$
\begin{gathered}
X_{1}=\partial_{2}, \quad X_{2}=\partial_{3}, \quad X_{3}=-\partial_{1}+x^{3} \partial_{2} \\
X_{4}=-x^{3} \partial_{1}+\frac{1}{2}\left(\left(x^{3}\right)^{2}-\left(x^{1}\right)^{2}\right) \partial_{2}+x^{1} \partial_{3}, \quad X_{5}=\partial_{4} .
\end{gathered}
$$

At each point $x_{0} \in M$ the isotropy algebra $\Gamma_{x_{0}}$ is spanned by

$$
Y_{x_{0}}=\frac{1}{2}\left(\left(x_{0}^{3}\right)^{2}+\left(x_{0}^{1}\right)^{2}\right) X_{1}-x_{0}^{1} X_{2}-x_{0}^{3} X_{3}+X_{4}
$$

By computing the flows of the vector fields (6.34) one can compute the corresponding connected group action $(\mu, G)$ on $\mathbb{R}^{4}$ with connected isotropy group $G_{x_{0}}$ generated by 
(6.35). The spacetime manifold is thus identified as the homogeneous space $\mathbb{R}^{4} \approx G / G_{x_{0}}$. Because both $G$ and $G_{x_{0}}$ are connected, the relevant computations for PSC can all be performed using its infinitesimal version in terms of $\Gamma$ and $\Gamma_{x_{0}}$. More generally, for any connected group action with connected isotropy whose infinitesimal generators are (6.34) and (6.35), respectively, our PSC results remain valid.

The general form of a $\Gamma$-invariant metric is given by

$$
\begin{aligned}
g(q)= & \frac{1}{2} q^{1}\left(d x^{1} \otimes d x^{1}+d x^{3} \otimes d x^{3}\right)+q^{2}\left(d x^{2}+x^{1} d x^{3}\right) \otimes\left(d x^{2}+x^{1} d x^{3}\right) \\
& +q^{3}\left(d x^{2} \odot d x^{4}+x^{1} d x^{3} \odot d x^{4}\right)+q^{4} d x^{4} \otimes d x^{4} \\
= & q^{i} h_{i}
\end{aligned}
$$

where $q^{i}$ are constants subject to

$$
\left(q^{1}\right)^{2}\left(4 q^{2} q^{4}-\left(q^{3}\right)^{2}\right)<0
$$

It is easily verified that the infinitesimal linear isotropy representation is, at each point, that of the Lorentz subalgebra of rotations. This guarantees that the infinitesimal form of the Palais condition is satisfied so that PSC2 is valid. The chain

$$
\chi=\partial_{1} \wedge \partial_{2} \wedge \partial_{3} \wedge \partial_{4}
$$

is $\Gamma$-invariant and satisfies $L_{\frac{\partial}{\partial x^{2}}} \chi=0$ so that, according to Proposition 5.4, a cochain map exists and PSC1 is valid. Thus PSC is valid for this group action.

The reduced Einstein tensor is of the form

$$
\mathcal{G}(g(q))=\mathcal{G}_{i}(q) f^{i}
$$

where

$$
f^{i}=\left(\partial_{1} \otimes \partial_{1}+\left(x^{1}\right)^{2} \partial_{2} \otimes \partial_{2}-2 x^{1} \partial_{2} \odot \partial_{3}+\partial_{3} \otimes \partial_{3}, \partial_{2} \otimes \partial_{2}, 2 \partial_{2} \odot \partial_{4}, \partial_{4} \otimes \partial_{4}\right)
$$

and

$$
\begin{gathered}
\mathcal{G}_{1}=-2 \frac{q^{2}}{\left(q^{1}\right)^{3}}, \\
\mathcal{G}_{2}=-2 \frac{6 q^{2} q^{4}-\left(q^{3}\right)^{2}}{\left(q^{1}\right)^{2}\left(\left(q^{3}\right)^{2}-4 q^{2} q^{4}\right)}, \\
\mathcal{G}_{3}=2 \frac{q^{2} q^{3}}{\left(q^{1}\right)^{2}\left(\left(q^{3}\right)^{2}-4 q^{2} q^{4}\right)},
\end{gathered}
$$




$$
\mathcal{G}_{4}=-4 \frac{\left(q^{2}\right)^{2}}{\left(q^{1}\right)^{2}\left(\left(q^{3}\right)^{2}-4 q^{2} q^{4}\right)} .
$$

We note that there are no $G$-invariant solutions to the vacuum Einstein equations $\mathcal{G}=0$ in this example. One can get equations that admit solutions by adding a $G$-invariant energy momentum tensor, which we won't bother to do. In any case, our goal here is to verify the equivalence of the reduced field equations with the Euler-Lagrange expressions coming from the reduced Lagrangian, which is guaranteed by the absence of the two obstructions to PSC. If we were viewing PSC as a statement about $G$-invariant critical points of an action functional, then we would have to admit that, in this case, there are no $G$-invariant critical points, which could be the case whether or not the reduced equations were in fact equivalent in the sense of Appendix B. This highlights a key difference between our definition of PSC and that of Palais in [11].

Using the cochain (6.36), the reduced Lagrangian is an ordinary function of the $q^{i}$ given by

$$
\hat{\lambda}(q)=-\frac{1}{2} \sqrt{\left(\left(q^{3}\right)^{2}-4 q^{2} q^{4}\right)} \frac{q^{2}}{q^{1}} .
$$

It is easily checked that the four Euler-Lagrange expressions associated with $\hat{\lambda}$ yield the 4 components of the Einstein tensor. In particular, we have that

$$
E_{i}(\hat{\lambda})=\frac{\partial \hat{\lambda}}{\partial q^{i}}=-\sqrt{-g(q)} \mathcal{G}_{i}(g(q)) .
$$

\section{Example 6.6b}

Finally we consider a transitive group action for which both PSC1 and PSC2 fail. The group $G$ with local coordinates $\lambda^{\mu}, \mu=1, \ldots, 5, \lambda^{\mu} \in \mathbb{R}$, acts on $\mathbb{R}^{4}$ with coordinates $x^{\alpha}$ by

$\mu(\lambda, x)=\left(e^{l \lambda^{5}} x^{1}-\frac{1}{2} e^{\epsilon \lambda^{5}}\left(\lambda^{4}\right)^{2} x^{2}-e^{k \lambda^{5}} \lambda^{4} x^{3}-\lambda^{3}, e^{\epsilon \lambda^{5}} x^{2}+\lambda^{1}, \lambda^{2}+e^{\epsilon \lambda^{5}} \lambda^{1} x^{2}+e^{k \lambda^{5}} x^{3}, x^{4}+\lambda^{5}\right)$ where $k, l$ and $\epsilon$ are parameters satisfying $2 k=l+\epsilon$. A basis for the Lie algebra of infinitesimal generators for this action is given by (33.29) in [15].

Let $x_{0}^{\alpha}=\left(x_{0}^{1}, x_{0}^{2}, x_{0}^{3}, x_{0}^{4}\right)$ be a point in $\mathbb{R}^{4}$, then the action of $G_{x_{0}}=\mathbb{R}$ is given by

$$
\mu^{\alpha}(\lambda, x)=\left(x^{1}-\frac{1}{2}\left(\lambda^{4}\right)^{2}\left(x^{2}-x_{0}^{2}\right)-\lambda^{4}\left(x^{3}-x_{0}^{3}\right), x^{2}, x^{3}+\lambda^{4}\left(x^{2}-x_{0}^{2}\right), x^{4}\right)
$$

where $\lambda^{4}$ is a coordinate for $G_{\mathbf{x}_{0}}$. The linear isotropy representation at the point $x_{0}^{\alpha}$ is given by the matrix

$$
\left(\begin{array}{cccc}
1 & -x_{0}^{2}\left(\lambda^{4}\right)^{2} & -\lambda^{4} & 0 \\
0 & 1 & 0 & 0 \\
0 & \lambda^{4} & 1 & 0 \\
0 & 0 & 0 & 1
\end{array}\right)
$$


Using the isotropy representation (6.38) in the constraint (2.4), the most general $G$ invariant metric is found to be

$$
\begin{aligned}
g= & q^{1}\left(e^{-2 k x^{4}} d x^{1} \odot d x^{2}+\frac{1}{2} e^{-2 k x^{4}} d x^{3} \otimes d x^{3}\right)+q^{2} e^{-2 \epsilon x^{4}} d x^{2} \otimes d x^{2} \\
& +q^{3} e^{-\epsilon x^{4}} d x^{2} \odot d x^{4}+q^{4} d x^{4} \otimes d x^{4} \\
= & q^{i} h_{i}
\end{aligned}
$$

where the $h_{i}, i=1, \ldots, 4$ are a basis for the $G$-invariant symmetric $\left(\begin{array}{l}0 \\ 2\end{array}\right)$ tensor fields.

It is easy to see from (6.38) that at each point $x \in M$ the vector $\left(\partial_{1}\right)_{x} \in\left(T_{x} M\right)^{G_{x}}$, and then from (6.39) that this vector is null with respect to all the invariant metrics. Therefore, by Proposition 5.6, the Palais condition is not satisfied for this group action and PSC2 fails.

Let us now consider PSC1. We shall see that PSC1 is valid if and only if $k=0$. It is easy to show that all $G$-invariant chains are proportional to

$$
\chi=e^{3 k x^{4}} \partial_{1} \wedge \partial_{2} \wedge \partial_{3} \wedge \partial_{4}
$$

The vector space of $G$-invariant vector fields is two-dimensional and is spanned by

$$
S_{1}=e^{l x^{4}} \partial_{1}, \quad S_{2}=\partial_{4}
$$

Since

$$
L_{S_{1}} \chi=0, \quad L_{S_{2}} \chi=3 k \chi
$$

we see that in accordance with Proposition 5.4 a cochain map exists if and only if $k=0$.

Let us consider the reduced Einstein equations with cosmological constant $\Lambda$ for the $G$-invariant spacetimes. We have that

$$
\mathcal{G}(g(q))+\Lambda g^{-1}(q)=\Delta_{i}(q) f^{i}
$$

where $f^{i}$ are a basis for the $G$-invariant symmetric $\left(\begin{array}{l}2 \\ 0\end{array}\right)$ tensor fields

$$
f^{i}=\left(\frac{4}{3} e^{2 k x^{4}} \partial_{1} \odot \partial_{2}+\frac{2}{3} e^{2 k x^{4}} \partial_{3} \otimes \partial_{3}, e^{2 l x^{4}} \partial_{1} \otimes \partial_{1}, 2 e^{l x^{4}} \partial_{1} \text { odot } \partial_{4}, \partial_{4} \otimes \partial_{4}\right) .
$$

and

$$
\begin{aligned}
& \Delta_{1}=\frac{9 k^{2}+3 \Lambda q^{4}}{q^{1} q^{4}} \\
& \Delta_{2}=-\frac{2 q^{2} q^{4}\left(l^{2}+l \epsilon+4 \epsilon^{2}\right)-3\left(q^{3}\right)^{2} k^{2}+\Lambda q^{4}\left(4 q^{2} q^{4}-\left(q^{3}\right)^{2}\right)}{4\left(q^{1} q^{4}\right)^{2}} \\
& \Delta_{3}=-\frac{q^{3}\left(3 k^{2}+\Lambda q^{4}\right)}{q^{1}\left(q^{4}\right)^{2}} \\
& \Delta_{4}=\frac{3 k^{2}+\Lambda q^{4}}{\left(q^{4}\right)^{2}} .
\end{aligned}
$$


Using the $G$-invariant chain $\chi$ in (6.40) to construct the reduced Lagrangian yields

$$
\hat{\lambda}(q)=-\sqrt{\frac{2\left(q^{1}\right)^{3}}{q^{4}}}\left(3 k^{2}+\frac{1}{2} \Lambda q^{4}\right) .
$$

Since $\hat{\lambda}$ is independent of $q^{2}$ and $q^{3}$ it is clear that two of the Euler-Lagrange expressions (now just partial derivatives) will be trivial:

$$
E_{2}(\hat{\lambda})=\frac{\partial \hat{\lambda}}{\partial q^{2}}=0, \quad E_{3}(\hat{\lambda})=\frac{\partial \hat{\lambda}}{\partial q^{3}}=0 .
$$

The corresponding field equation components are $\Delta_{2}$ and $\Delta_{3}$, both of which are non-zero. This is how the failure of PSC2 manifests itself. The remaining Euler-Lagrange expressions are

$$
E_{1}(\hat{\lambda})=\frac{\partial \hat{\lambda}}{\partial q^{1}}=-3 \sqrt{\frac{1}{2\left(q^{1}\right)^{3} q^{4}}}\left(3 k^{2}+\frac{1}{2} \Lambda q^{4}\right)
$$

and

$$
E_{4}(\hat{\lambda})=\frac{\partial \hat{\lambda}}{\partial q^{4}}=-\sqrt{\frac{1}{2 q^{1}\left(q^{4}\right)^{3}}}\left(-3 k^{2}+\frac{1}{2} \Lambda q^{4}\right) .
$$

By comparing (6.41) with $\Delta_{1}$ and (6.42) with $\Delta_{4}$ it is clear that these Euler-Lagrange

expression of $\hat{\lambda}$ agree with the corresponding terms in the field equations if and only if $k=0$, which is the case where PSC1 holds.

\section{Acknowledgments}

We would like to thank Ian Anderson for essential contributions to many aspects of the problems considered here. This work was supported in part by NSF grants DMS-9804833, PHY-9732636 and PHY-0070867 to Utah State University.

\section{Appendix A: Additional fields beyond the metric}

Much of the preceding analysis can be generalized to a gravitational field theory involving a metric coupled to matter fields, or to a matter field theory propagating on a fixed spacetime. We briefly indicate here how this generalization goes (see also [14]).

Let us collectively denote all dynamical fields on a spacetime manifold $M$ using an abstract symbol $\varphi$. We assume that all fields can be viewed as sections of fiber bundles $E \rightarrow M$. If the theory is that of a gravitational field coupled to matter then $\varphi$ would 
include the metric, scalar fields, Maxwell fields, etc. If the theory is a field theory on a fixed background spacetime $(M, g)$, then $\varphi$ includes just the dynamical fields. We assume that there is given a group action of $G$ on $M$ with a specified lift to the space of fields $\varphi$ obeying the regularity conditions of [13], and that there exist $G$-invariant fields $\varphi(\hat{q})$ with respect to that group action. Here we denote by $\hat{q}$ the fields on $M / G$ that parametrize the space of $G$-invariant fields on $M$. For more details about all these constructions, see [13].

Let $\lambda$ be a $G$-equivariant Lagrangian for the fields $\varphi$. The first variational formula

$$
\delta \lambda=E(\lambda) \cdot \delta \varphi+d \eta(\delta \varphi)
$$

defines $\eta$ up to an exact form. We assume that the $(n-1)$-form $\eta$ can be chosen to be $G$-equivariant. For a generally covariant theory of gravity coupled to matter fields this is guaranteed [18]. If the metric is fixed, i.e., we consider matter fields on a fixed spacetime, then this will again be guaranteed provided chosen symmetry group acts by isometries [20]. We would normally need this latter assumption in any case in order to render the matter Lagrangian $G$-equivariant.

With this assumption in hand, we can repeat the analysis of sections 4 and 5, with very similar results. As in $\S 4$ we can define field equations, reduced field equations and the reduced Lagrangian associated with the $G$ action. As in $\S 5$ we can obtain the two conditions (PSC1 and PSC2) for PSC. The condition PSC1 is described in terms of the Lie algebra cohomology exactly as before: $\mathcal{H}^{l}\left(\Gamma, G_{x}\right) \neq 0$ for all $x \in M$, where $l$ is the dimension of the group orbits in $M$. Thus PSC1 is insensitive to the field content of the theory. On the other hand, the validity of PSC2 depends upon the way in which the isotropy group $G_{x}$ is represented on $S_{x}$, the vector space of values of the field variations $\delta \varphi$ at each $x \in M$. As in $\S 5$, denote by $S_{x}^{G x}$ the $G_{x}$-invariant points in $S_{x}$ and denote by $\left(S_{x}^{*}\right)^{G_{x}}$ the $G_{x}$-invariant points in $S_{x}^{*}$. Denote by $\left(S_{x}^{G x}\right)^{0} \subset S_{x}^{*}$ the annihilator of $S_{x}^{G x}$. As before, PSC2 is satisfied if and only if

$$
\left(S_{x}^{*}\right)^{G_{x}} \cap\left(S_{x}^{G_{x}}\right)^{0}=0, \quad \forall x \in M
$$

A couple of examples of (A.1) are worth mentioning. For a theory involving scalar fields coupled to gravity, so that $\varphi$ includes a metric and some scalar fields, the action of $G_{x}$ on the scalar fields is trivial and so the condition (A.1) reduces to that studied in $\S 5$ on the metric alone. So, for example, in four dimensions with connected isotropy groups only the null rotation subgroups lead to a failure of PSC2 for a metric coupled to scalar fields. For scalar fields propagating on a fixed ( $G$-invariant) background spacetime the action of $G_{x}$ on $S_{x}$ is completely trivial so that

$$
S_{x}^{G_{x}}=S_{x}
$$


and it follows that (A.1) is always satisfied. As another example, suppose that $\varphi$ includes a Maxwell field and a metric, or just a Maxwell field propagating on a fixed spacetime. For simplicity, let us assume that the $G$-action on the Maxwell field does not include any gauge transformations, that is, a $G$-invariant Maxwell field is just a $G$-invariant 1-form on spacetime. It is not hard to see that the condition (A.1) leads to the null rotation subgroups as the only isotropy groups that fail to satisfy PSC2.

\section{Appendix B: Equivalence of differential equations}

For our purposes we will define "equivalent" differential equations as follows. Let $\Delta_{1}$ and $\Delta_{2}$ be two differential operators acting on a given space of fields $\mathcal{M}$. We view them as maps :

$$
\Delta_{1}: \mathcal{M} \rightarrow V_{1}, \quad \Delta_{2}: \mathcal{M} \rightarrow V_{2},
$$

where $V_{1}$ and $V_{2}$ are vector spaces. We say that the differential equations $\Delta_{1}=0$ and $\Delta_{2}=0$ are equivalent if there is a smooth map

$$
H: \mathcal{M} \times V_{1} \rightarrow V_{2},
$$

locally constructed from the fields $\varphi \in \mathcal{M}$, such that for each $\varphi$

$$
\Delta_{2}(\varphi)=H(\varphi) \cdot \Delta_{1}(\varphi),
$$

with $H(\varphi): V_{1} \rightarrow V_{2}$ an isomorphism. In this case we write

$$
\Delta_{1}=0 \Longleftrightarrow \Delta_{2}=0
$$

The equivalence of equations shown in (4.1) is of the type just described.

The reduced differential operator $\hat{\Delta}$, described in $\S 3$, can be viewed as a mapping

$$
\hat{\Delta}: \hat{\mathcal{Q}} \rightarrow V
$$

where $V$ is a vector space. Likewise,

$$
E(\hat{\lambda}): \hat{\mathcal{Q}} \rightarrow W,
$$

where elements of $W$ differ from those of $V$ by a tensor product with a volume form on $M / G$. The vector spaces $V$ and $W$ are isomorphic. PSC asserts that

$$
E(\hat{\lambda})=0 \Longleftrightarrow \hat{\Delta}=0 .
$$

\section{References}

1. S. Hawking, Mon. Not. R. Astron. Soc. 142, 129 (1969). 
2. M. MacCallum and A. Taub, Commun. Math. Phys. 25, 173 (1972).

3. M. Ryan, J. Math. Phys. 15, 812 (1974).

4. G. Sneddon, J. Phys. A 9, 229 (1976).

5. M. MacCallum in General Relativity: An Einstein Centenary Survey, edited by S. Hawking and W. Israel (Cambridge University Press, Cambridge 1979).

6. R. T. Jantzen, Nuovo Cim. 55B, 161-172 (1980); Phys. Rev. D 34, 424-433 (1986); Phys. Rev. D 35, 2034-2035 (1987).

7. A. Ashtekar and J. Samuel, Class. Quantum Grav. 8, 2191 (1991).

8. J. Pons and L. Shepley, Phys. Rev. D 58, 024001 (1998).

9. W. Pauli, Relativitätstheorie, Encyclopedia der Matematicshcen Wissenschaftern, Vol. 19, B.G. Teubner, Leipzig, 1921.

10. D. Lovelock, Nuov. Cim. B73, 260 (1973).

11. R. Palais, Commun. Math. Phys. 69, 19-30 (1979).

12. I. M. Anderson and M. E. Fels, Amer. J. Math. 119, 609 (1997).

13. I. M. Anderson, M. E. Fels, and C. G. Torre, Commun. Math. Phys. 212, 653-686 (2000).

14. I. M. Anderson, M. E. Fels, C. G. Torre, "Group Invariant Solutions without Transversality and the Principle of Symmetric Criticality", preprint math-ph/9910014.

15. A. Petrov, Einstein Spaces, (Pergamon Press, 1969).

16. I. M. Anderson and M. E. Fels, "Transverse group actions on bundles", to appear in Topology and its Applications, 2001.

17. R. Coquereaux and A. Jadczyk, Riemannian Geometry, Fiber Bundles, Kaluza-Klein Theories and all that, Lecture Notes in Physics 16 (World Scientific, Singapore, 1988).

18. V. Iyer and R. Wald, Phys. Rev. D 50, 846-864 (1994).

19. P. Olver, Applications of Lie Groups to Differential Equations, (Springer-Verlag, New York 1993).

20. I. M. Anderson, M. E. Fels, C. G. Torre, unpublished.

21. J. Patera and P. Winternitz, J. Math. Phys. 16, 1597 (1975). 
22. D. Kramer, H. Stephani, E. Herlt, M. MacCallum, Exact Solutions of Einstein's Field Equations, edited by E. Schmutzer (Cambridge University Press, Cambridge, 1980).

23. J. D. Romano and C. G. Torre, Phys. Rev. D 53, 5634 (1996). 